Revue des patrimoines

\title{
Les fac-similés de la tombe égyptienne de Sennefer : une solution pour la conservation de l'original?
}

Laëtitia Maggio

\section{OpenEdition}

Journals

Édition électronique

URL : http://journals.openedition.org/insitu/10693

DOI : 10.4000/insitu. 10693

ISSN : 1630-7305

Éditeur

Ministère de la culture

Référence électronique

Laëtitia Maggio, «Les fac-similés de la tombe égyptienne de Sennefer : une solution pour la

conservation de l'original ? », In Situ [En ligne], 22 | 2013, mis en ligne le 15 novembre 2013, consulté le 01 mai 2019. URL : http://journals.openedition.org/insitu/10693; DOI : 10.4000/insitu.10693

Ce document a été généré automatiquement le 1 mai 2019.

\section{(c)}

In Situ Revues des patrimoines est mis à disposition selon les termes de la licence Creative Commons Attribution - Pas d'Utilisation Commerciale - Pas de Modification 4.0 International. 


\title{
Les fac-similés de la tombe égyptienne de Sennefer : une solution pour la conservation de l'original?
}

\author{
Laëtitia Maggio
}

Nous remercions vivement : Étienne Féau (conservateur en chef, Ministère de la Culture), Jean-Luc Bovot (ingénieur d'études, Département des Antiquités égyptiennes, musée du Louvre), Françoise Chaserant (conservateur en chef, musée de Tessé au Mans), Laurent Bavay (professeur à l'Université libre de Bruxelles, directeur de la Mission archéologique de la Nécropole thébaine MANT), Aude Aussilloux (restauratrice), Jean-Jacques Ezrati (ingénieur, C2RMF), Bettina Schmitz (conservateur) et Dorothea Lindemann (restauratrice, musée Roemer et Pelizaeus d'Hildesheim), Alain Cunillera (dirigeant de la société Basic Théâtral), Paulette Hugon (ingénieur au Laboratoire de Recherche des Monuments Historiques).

Communication présentée lors des journées d'études consacrées à l'Actualité de la recherche en peinture murale, organisées par le musée des Monuments français les 25 et 26 octobre 2011, à la

Cité de l'architecture et du patrimoine.

Les menaces qui pèsent sur les décors de monuments antiques sont préoccupantes et concilier conservation et exposition est rarement possible. Les tombes égyptiennes sont au cœur de cette problématique puisque sur la rive gauche du Nil, des monuments vieux de plus de 3000 ans sont en danger. Les conditions naturelles difficiles, les réutilisations et occupations multiples souvent peu respectueuses, les pillages, le vandalisme ainsi que le tourisme de masse ont contribué à accélérer un processus de dégradation irréversible. Les tombes thébaines, et en particulier celles des nobles, n'échappent pas à ce constat et la conservation de leurs peintures murales est un sujet de préoccupation pour les spécialistes. En 1979, tout le secteur thébain a été inscrit sur la liste du patrimoine mondial de l'UNESCO <lien vers http://whc.unesco.org/fr/list/87/>. Les tombes thébaines 
ont alors été reconnues comme objets culturels et œuvres d'art d'un grand intérêt, ce qui a permis une prise de conscience générale de l'urgence de leur sauvegarde (fig. $\mathbf{n}^{\circ} \mathbf{1}$ ).

Figure 1

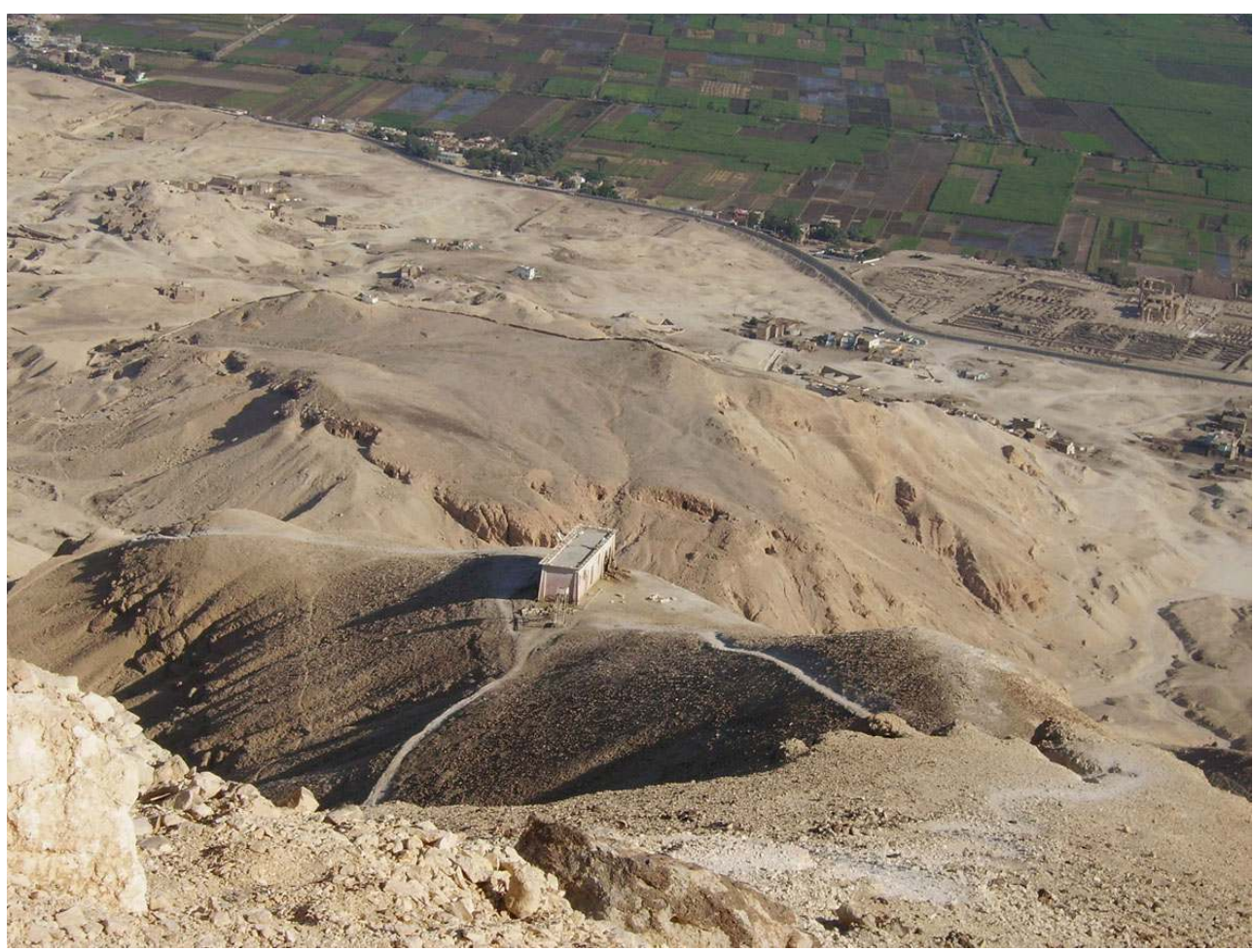

Vue de la rive ouest de Thèbes depuis la montagne thébaine, colline de Cheikh Abd el-Gourna.

Phot. Maggio, Laëtitia, 2008. (c) Laëtitia Maggio.

2 Aménagée sur le flanc sud de la colline de Cheikh Abd el-Gourna, la tombe de Sennefer (TT 96) fait partie de cet ensemble exceptionnel menacé (fig. $\mathbf{n}^{\circ} \mathbf{2}$ ). Celle qu'on surnomme la « tombe aux vignes », en raison des treilles qui recouvrent partiellement les plafonds de son caveau, a été conçue pour Sennefer, Prince de la Ville du Sud (Thèbes), intendant des jardins et des greniers d'Amon, sous le règne d'Amenhotep II à la XVIII ${ }^{\mathrm{e}}$ dynastie (vers 1427-1401 avant J.-C.). Son caveau (TT 96B) entièrement peint la distingue des autres tombes (fig. $\mathbf{n}^{\circ} \mathbf{3}$ ). L'état de conservation remarquable de ses peintures et l'originalité de l'iconographie font du caveau de Sennefer l'un des plus exceptionnels de la nécropole. Il est également l'un des seuls monuments de la Vallée des Nobles à avoir été entièrement enregistré et à avoir fait l'objet d'une reconstitution grandeur nature. Le rôle de ces reproductions face aux œuvres originales présente un grand intérêt dans un contexte de conservation préventive. Le remplacement de la visite de la tombe originale par celle de son substitut pourrait ainsi résoudre le dilemme entre exposition et conservation. 
Figure 2

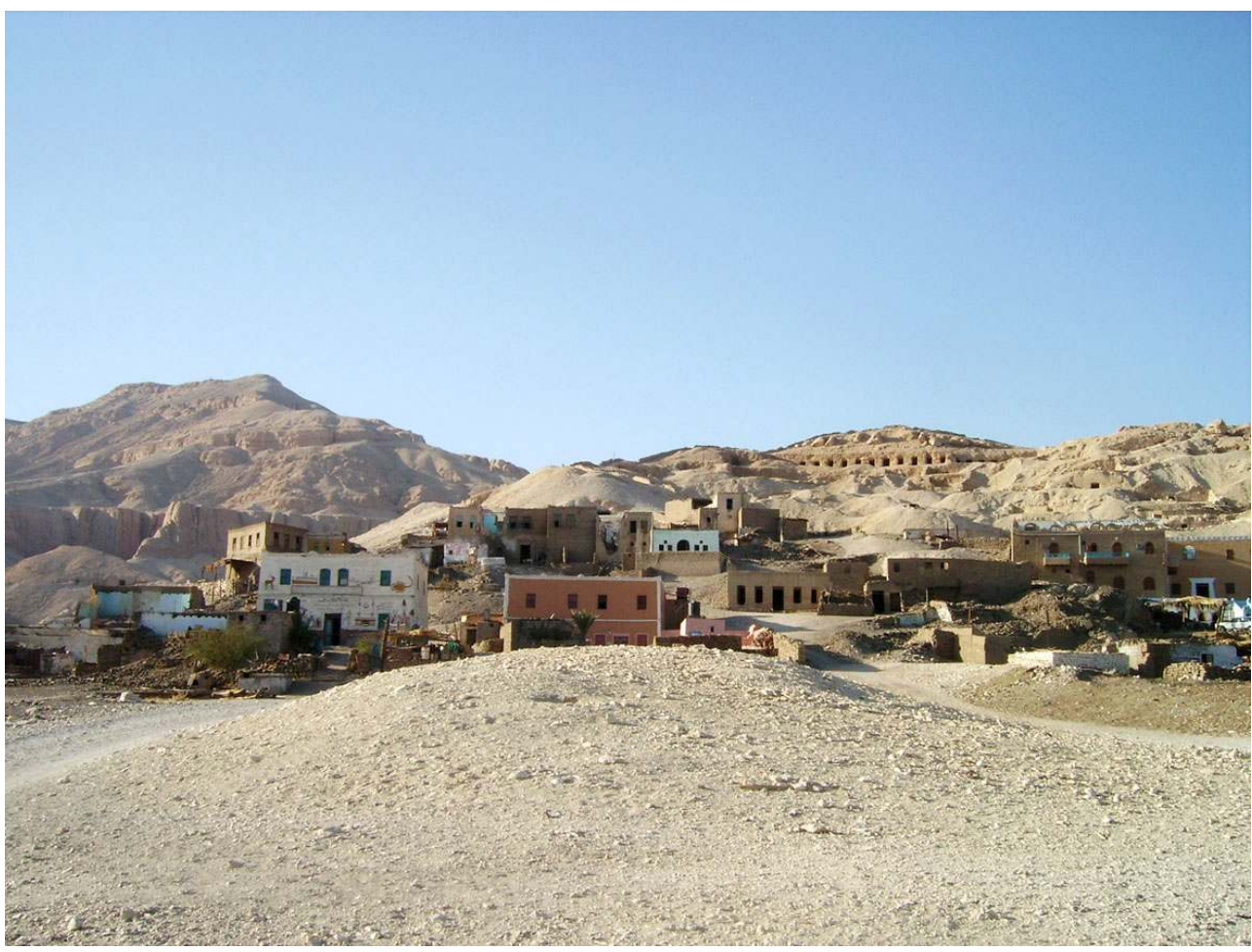

La colline de Cheikh Abd el-Gourna, le village implanté sur la nécropole antique.

Phot. Maggio, Laëtitia, 2008. (c) Laëtitia Maggio. 
Figure 3

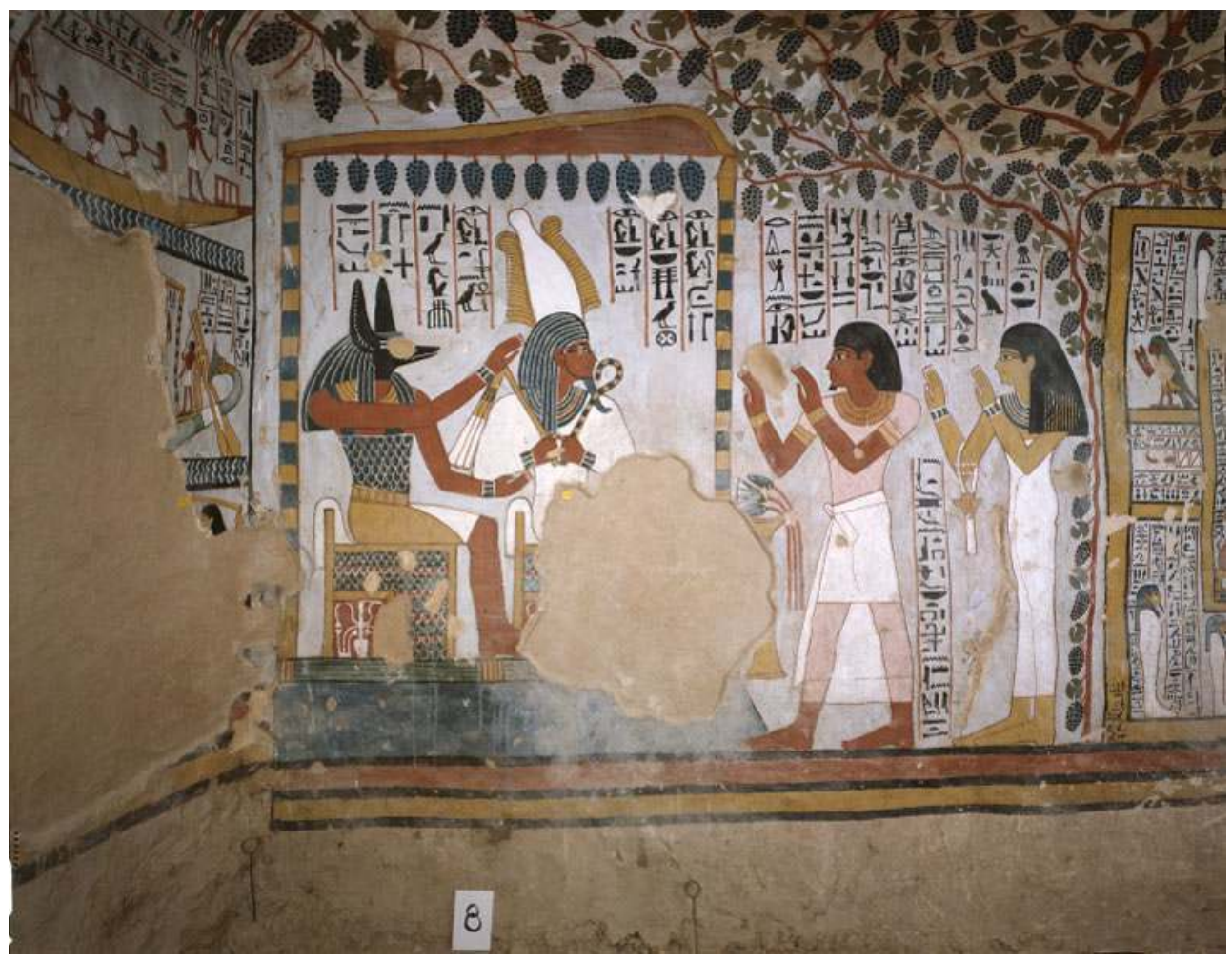

Scène du caveau de la tombe de Sennefer (TT 96B).

Phot. Bovot, Jean-Luc, 1984. (c) Fondation Kodak Pathé.

La sauvegarde de l'original : l'état de conservation actuel de la tombe de Sennefer et les perspectives

Description du monument et des techniques employées 


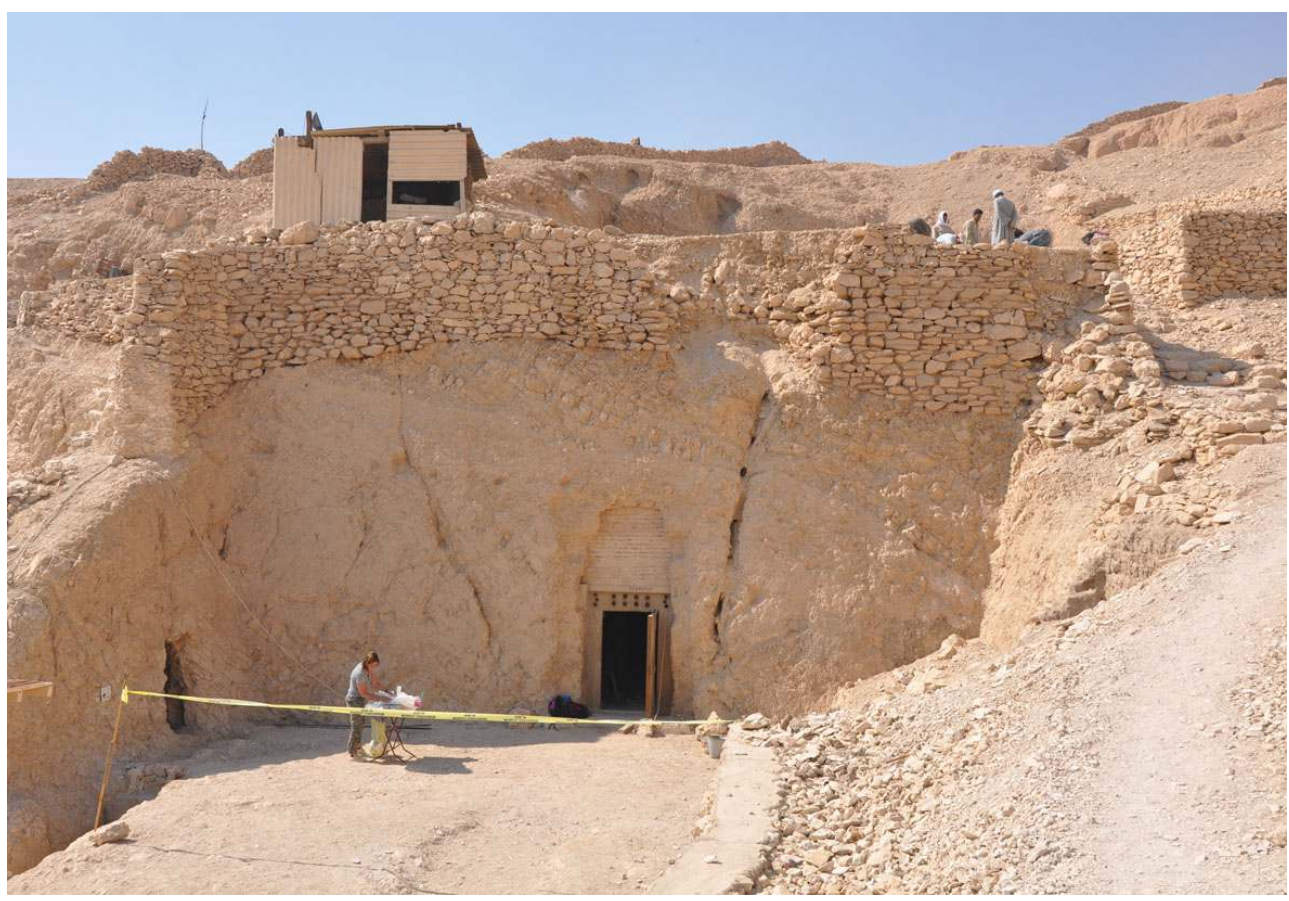

La cour de la tombe de Sennefer : entrée de la chapelle au fond et accès au caveau à gauche.

Phot. Bavay, Laurent, 2011. (C) ULB - MANT.

3 La tombe de Sennefer est construite sur le plan classique des tombes du Nouvel Empire associant une chapelle en T inversé (TT 96A) et un caveau souterrain (TT 96B). Chacun possède une entrée propre, le couloir d'accès du caveau débouchant dans l'angle sud de la cour précédant la façade de la chapelle (fig. $\left.\mathbf{n}^{\circ} \mathbf{4}\right)$. Une tombe thébaine est d'ordinaire constituée d'une structure à la surface, appelée chapelle, décorée et accessible aux vivants pour le culte funéraire du défunt, et d'un caveau creusé dans le sol contenant la momie et le mobilier funéraire. Le caveau est donc généralement inaccessible aux vivants et non décoré. Celui de la tombe de Sennefer se présente sous la forme d'une pièce à peu près carrée de 7,50 mètres de long sur 6,70 mètres de large, aux parois bosselées et au plafond soutenu par quatre piliers. La hauteur varie de 2,20 mètres à 2,80 mètres. Cette chambre funéraire est précédée d'une antichambre au plafond bas (fig. $\mathbf{n}^{\circ} 5$ ). 


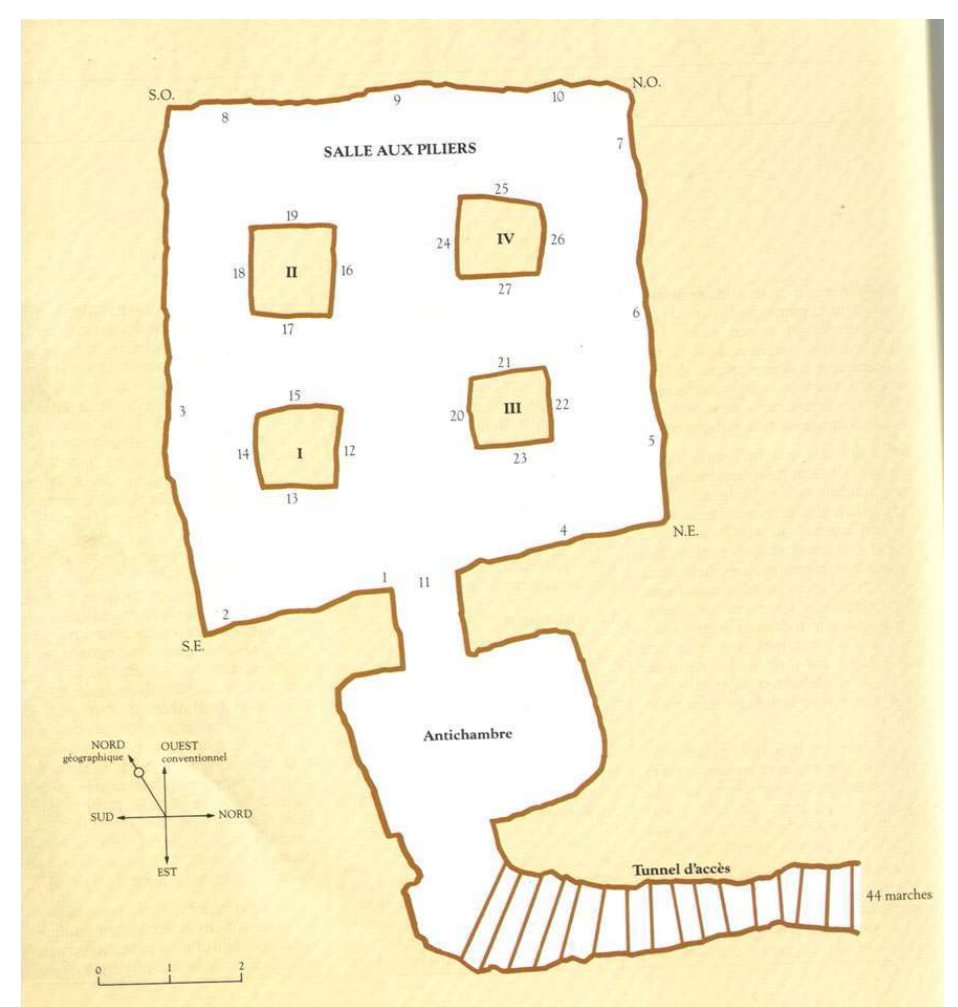

Plan du caveau de la tombe de Sennefer (TT 96B), publié dans COLLECTIF. Reconstitution du caveau de Sennefer dit «Tombe aux vignes »: Thèbes-Ouest, Cheikh Abd el Gournah, XVIIle dynastie. Paris : Fondation Kodak Pathé, 1985, p. 28.

Repro. Kurz, Marcel, 1985. (c) Fondation Kodak Pathé.

4 Les techniques employées pour l'exécution des peintures murales de la chapelle et du caveau de la tombe de Sennefer sont similaires ${ }^{1}$. L'édifice étant creusé dans la montagne thébaine, le lissage des parois a été effectué à l'aide d'une préparation composée d'enduits de terre (moûna, mélange de limon, de paille et de résidus organiques) pour homogénéiser grossièrement la surface, puis d'enduits de gypse pour obtenir des couches plus fines. La couche finale d'enduit est très mince, lisse et d'un ton moyen sur laquelle un lait de chaux gris bleuté pâle a été passé. Les formes sont dessinées au trait rouge, puis les couleurs sont appliquées. Les peintures sont des détrempes sur enduit sec, les pigments étant liés par une solution de gomme arabique ${ }^{2}$.

\section{Les facteurs de dégradation}

5 La connaissance de l'état d'altération des enduits et des peintures murales ainsi que des causes des dégradations est fondamentale pour toute entreprise de conservationrestauration. Les témoignages anciens des premiers égyptologues qui ont pénétré dans la tombe au début du XIX siècle, bien que rares et approximatifs, permettent de différencier les altérations les plus récentes des plus anciennes. Caveau et chapelle ont été inégalement étudiés ${ }^{3}$. Par ailleurs, leur histoire est différente, ils n'ont donc pas subi les mêmes dégradations. Ils font cependant partie d'un même ensemble et la technique d'exécution des peintures murales est similaire. Des risques communs, en particulier d'ordre naturel, pèsent donc à la fois sur le caveau et sur la chapelle. 
Les travaux de la Mission archéologique dans la Nécropole thébaine de l'Université libre de Bruxelles <lien vers http://crea.ulb.ac.be/Thebes.html> ont permis d'identifier les facteurs de dégradation des peintures murales de la chapelle, qui peuvent être appliqués au caveau. Certaines altérations ont pour origine la nature-même de la roche dans laquelle est creusée la tombe. Le calcaire est d'assez mauvaise qualité à cet emplacement, les veines marneuses friables étant nombreuses. Les ouvriers ont dû connaître des difficultés pour tailler des parois régulières, d'où l'emploi systématique d'enduits et de badigeons. Ces défauts structurels de la roche ont entraîné des effondrements, des pertes d'adhérence des enduits sur la paroi et l'apparition de fentes ou de fissures ${ }^{4}$ (fig. $\left.\mathbf{n}^{\circ} \mathbf{6}\right)$. Ces dégradations sont particulièrement apparentes dans la chapelle, mais certaines lacunes à l'intérieur du caveau sont aussi la conséquence de ce support médiocre. De qualité très inégale, il engendre des comportements mécaniques différentiels à l'origine de soulèvements de matière ou de clivages. La mise en œuvre de la tombe est également problématique, ainsi que l'hétérogénéité des matériaux qui composent les enduits, leur dosage et la façon dont ils ont séché. Tout ceci entraîne des pertes d'adhérence entre les différentes strates et, avec les reliefs superficiels, des pertes de cohésion et des fissures (fig. $\mathbf{n}^{\circ}$ ). Le liant employé a perdu également ses qualités avec le temps causant des craquelures et des pulvérulences de matière ${ }^{5}$.

Figure 6

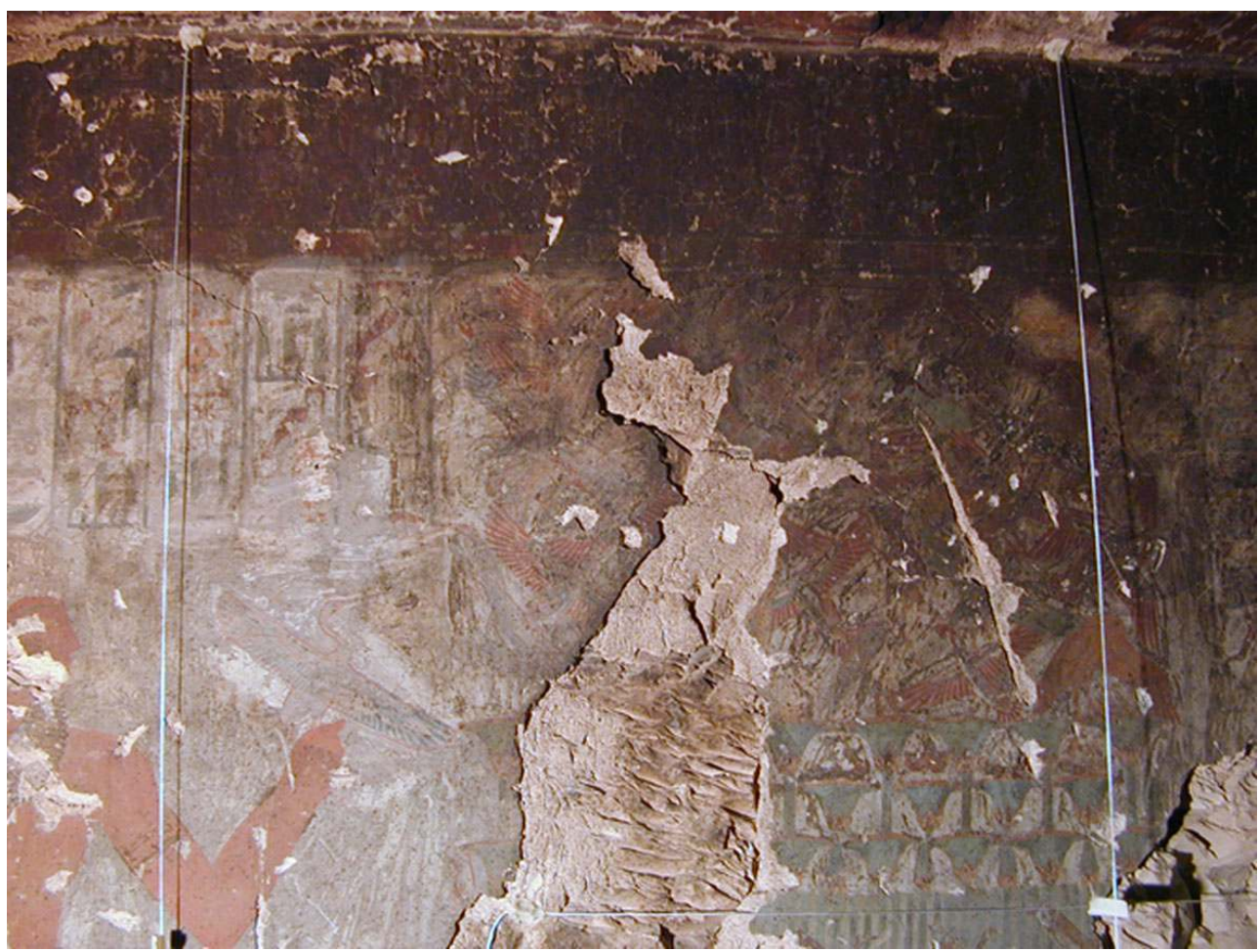

Chapelle de la tombe de Sennefer (TT 96A) : importantes lacunes et soulèvements de toutes les strates d'enduits.

Phot. Aussilloux, Aude, 2001. (c) ULB - MANT. 


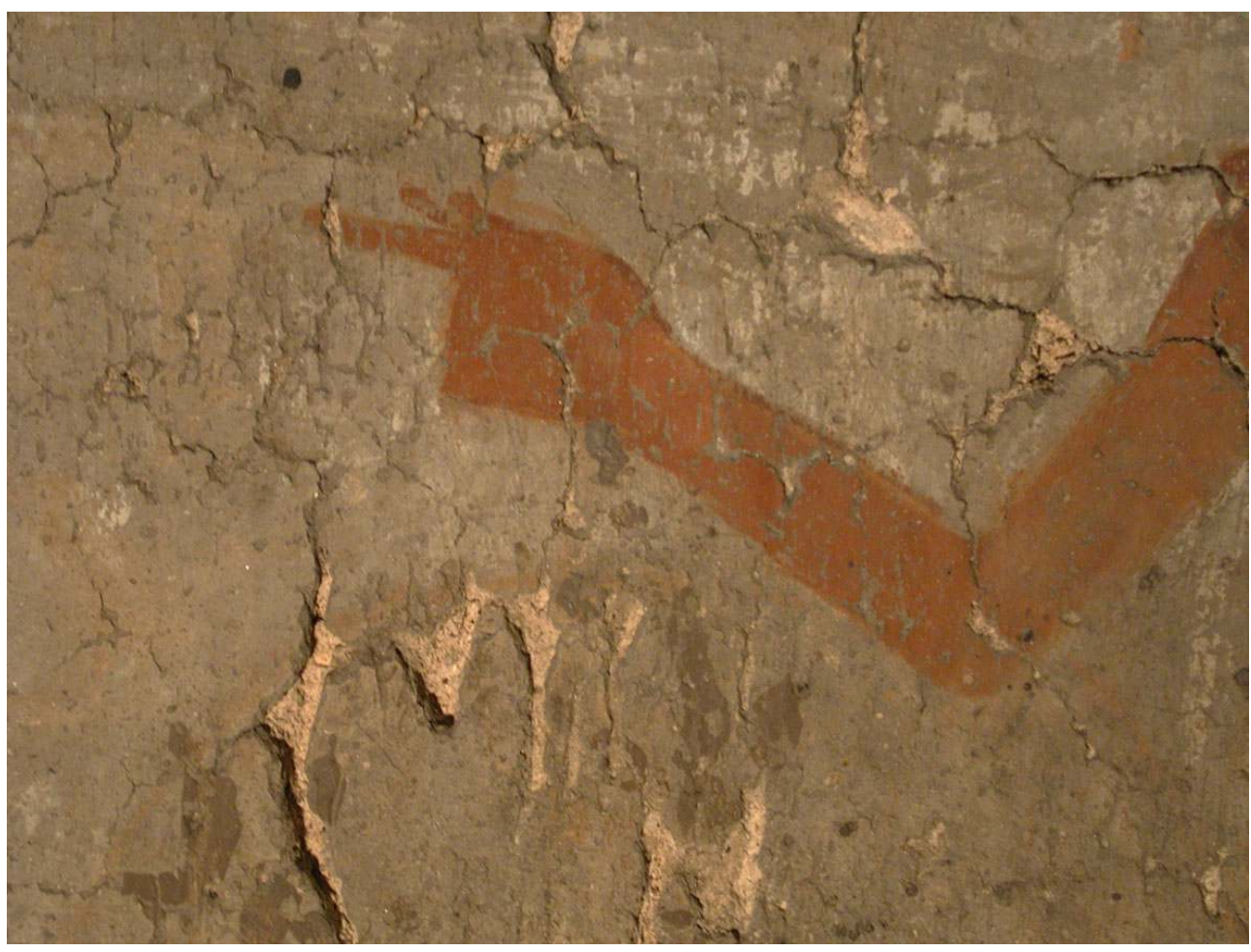

Chapelle de la tombe de Sennefer (TT 96A) : fissures fines, craquelures et perte de matière des enduits peints.

Phot. Aussilloux, Aude, 2001. (c) ULB - MANT.

7 Les effondrements et fissures sont quelquefois dus aux accidents climatiques et géologiques qui ont eu et ont encore lieu dans cette zone de l'Égypte, fortes pluies ou tremblements de terre. Plus encore que le climat extérieur, ce sont les conditions microclimatiques à l'intérieur de la tombe qui sont déterminantes pour la cohérence et l'adhérence des enduits. Les variations brusques de température et d'humidité constituent la principale menace pour les peintures murales. Le caveau de la tombe de Sennefer bénéficie certes naturellement d'une grande stabilité micro-climatique malgré les fluctuations extérieures, mais la création de conditions climatiques artificielles menace les peintures. L'impact des visiteurs sur le climat a déjà été mesuré pour la tombe de Néfertari, dans la nécropole voisine de la Vallée des Reines, et les résultats sont éloquents ${ }^{6}$. Les variations de température et d'hygrométrie sont probablement même amplifiées pour la tombe de Sennefer puisque son volume est plus réduit que celle de Néfertari, $90 \mathrm{~m}^{3}$ contre $250 \mathrm{~m}^{3}$. Ces variations brusques provoquent des tensions internes et des décollements ou craquelures, en raison d'un écart de coefficients d'expansion entre le liant des peintures et l'enduit?

Les visiteurs sont à l'origine d'autres facteurs de dégradations. L'éclairage engendre de la chaleur qui dessèche la gomme d'acacia. Une condensation peut aussi se créer sur les parois et s'y accroche lorsqu'elle est mêlée à la poussière, sous forme de croûtes ancrées aux décors muraux ${ }^{8}$. L'empoussièrement des parois, aggravé par le piétinement des visiteurs, était encore plus important avant la mise en place d'un plancher au début des années 1990 puisque le sol du caveau était nu, composé de terre battue (fig. $\left.\mathbf{n}^{\circ} \mathbf{8}\right)$. La poussière adhère d'autant mieux aux parois du caveau qu'elles sont rugueuses et 
souffrent d'infiltrations salines causant des boursouflures. Outre l'aspect esthétique, cette poussière menace la conservation des décors car elle favorise le développement de microorganismes sur les parois.

Figure 8

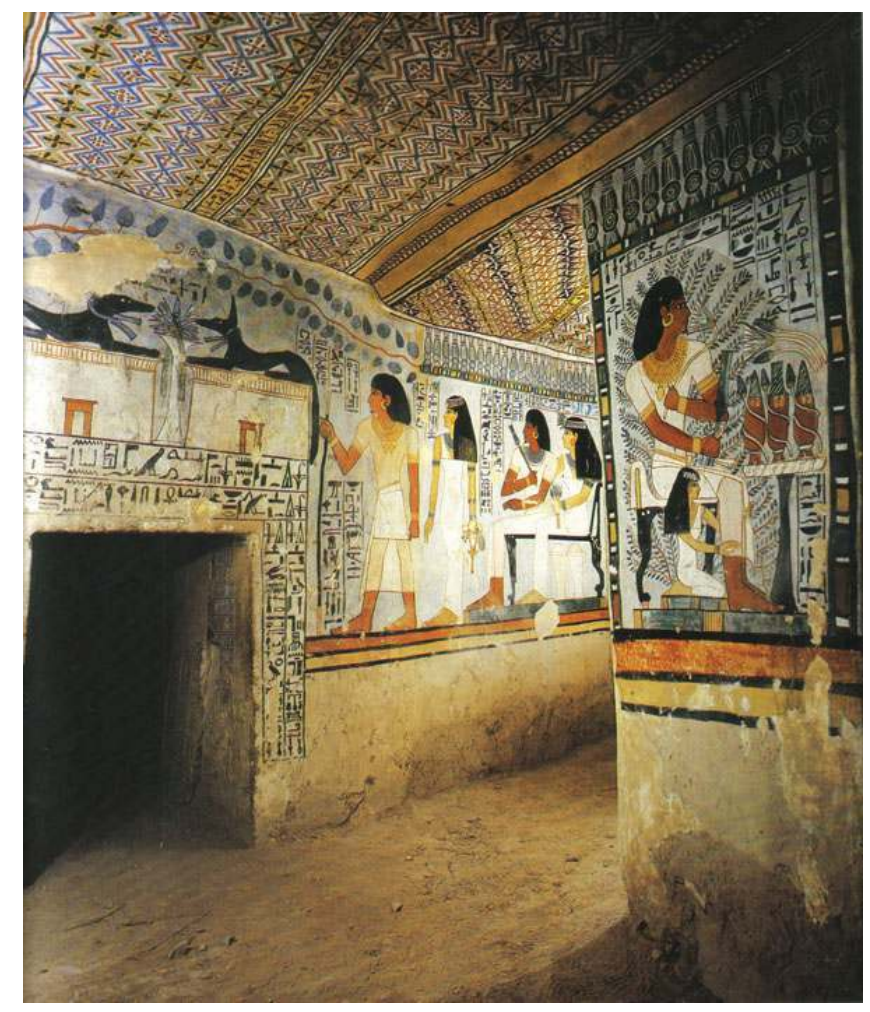

Vue du caveau de la tombe de Sennefer (TT 96B) avec son sol en terre battue, publiée dans COLLECTIF. Reconstitution du caveau de Sennefer dit « Tombe aux vignes »: Thèbes-Ouest, Cheikh Abd el Gournah, XVIIle dynastie. Paris : Fondation Kodak Pathé, 1985, p. 33.

Repro. Bovot, Jean-Luc, 1985. (c) Fondation Kodak Pathé.

Le caveau pose également des problèmes spécifiques dus à son utilisation. Il a été dès sa découverte dans la première moitié du XIX ${ }^{\mathrm{e}}$ siècle ouvert au public et cette ouverture a obligé les autorités à prendre des mesures favorables au monument, entretien, surveillance et gardiennage. Considéré comme monument historique, il n'a pas eu d'autres utilisations que le tourisme ou l'étude scientifique. Le caveau échappe donc à la plupart des dégradations intentionnelles dont a souffert la chapelle ${ }^{9}$. Les pilleurs anciens ou modernes l'ont cependant altéré. La plupart des mutilations des parois sud et ouest sont anciennes et sont le fait de voleurs « en quête d'amulettes ou d'une communication éventuelle avec d'autres caveaux $»^{10}$. D'autres dégradations ont été effectuées après 1900 , en particulier, le prélèvement d'une partie des décors par des collectionneurs voulant rapporter un souvenir de leur voyage, pratique courante en Égypte. Une partie du décor a disparu entre la découverte de la tombe et aujourd'hui ${ }^{11}\left(\right.$ fig. $\left.\mathbf{n}^{\circ} \mathbf{9}\right)\left(\right.$ fig. $\left.\mathbf{n}^{\circ}{ }^{10}\right)$. 
Figure 9

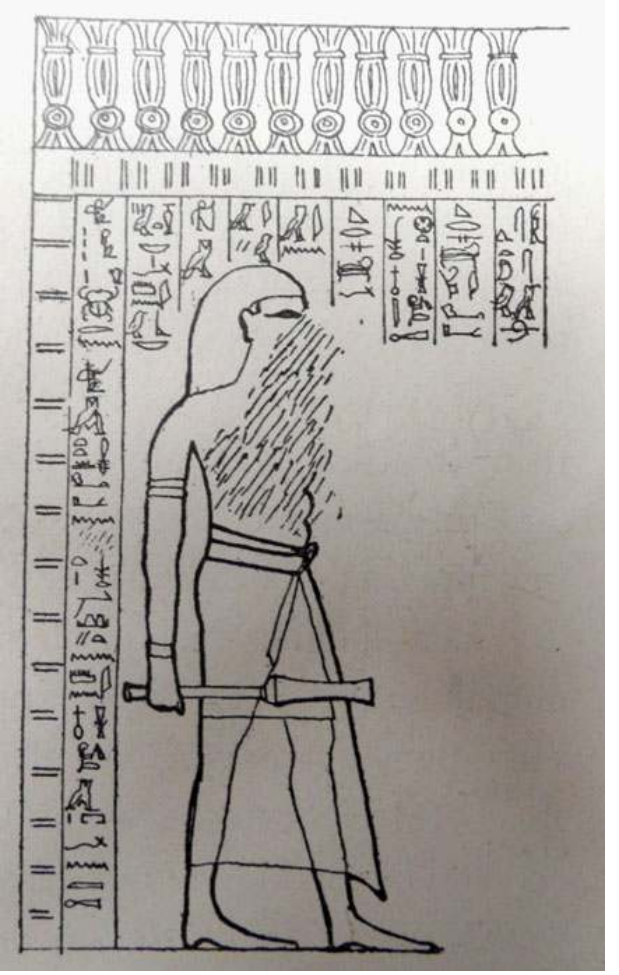

Dessin d'une scène du caveau de la tombe de Sennefer (TT 96B), publié dans VIREY, Philippe. « La tombe des vignes à Thèbes ». RT Recueil de travaux relatifs à la philologie et à l'archéologie, 1898, $n^{\circ} 20$. Repro. Virey, Philippe, 1898. (c) Philippe Virey. 


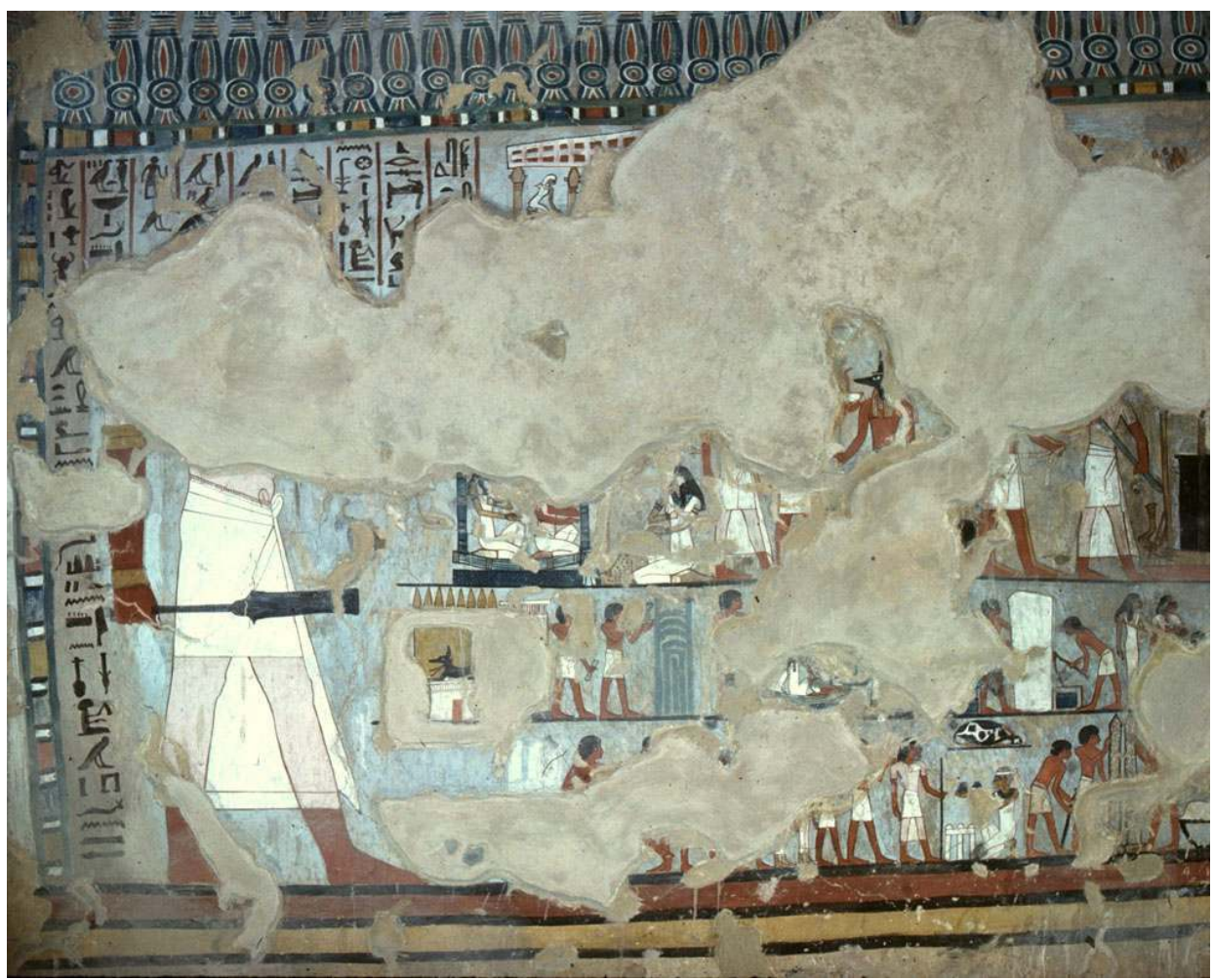

SCÈNE du CAVEAU de LA TOMBE de SENNEFER (Tt 96B).

Phot. Bovot, Jean-Luc, 1984. (c) Fondation Kodak Pathé.

\section{Les solutions de conservation préventive envisagées et envisageables}

Pour lutter contre ces différents facteurs de dégradations, les autorités égyptiennes ont décidé d'agir dès les années 1980. L'action a été différente pour la chapelle qui avait besoin d'une campagne importante de conservation-restauration ${ }^{12}$ et le caveau qui nécessitait une prise de mesures immédiate pour limiter les dégâts dus au tourisme.

Depuis le vidage de la tombe de Sennefer par Robert Mond en 1902-1903 et son ouverture au public, rien n'avait été fait pour limiter les dégradations dues aux visiteurs, hormis le gardiennage. Une première étape a été franchie en 1984 lorsque, pour établir une couverture photographique dans les meilleures conditions possibles en vue de la réalisation d'un fac-similé, un nettoyage du caveau a été effectué par le Service des Antiquités égyptiennes ${ }^{13}$. Au début des années 1990, il a pris la décision de poser un plancher de bois et des vitres en plexiglas maintenues par des cadres en bois et métal devant les parois pour prévenir l'empoussièrement et les risques mécaniques liés aux visiteurs $^{14}$ (fig. $\left.\mathbf{n}^{\circ} \mathbf{1 1}\right)$ (fig. $\mathbf{n}^{\circ} \mathbf{1 2}$ ). 


\section{Figure 11}

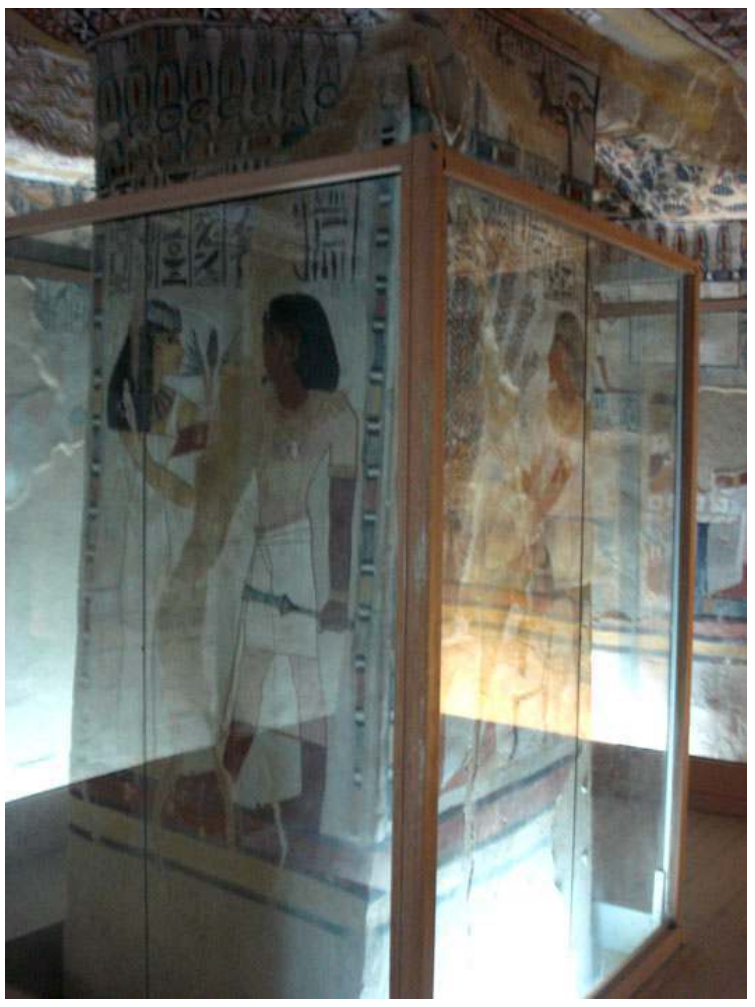

Vue d'un pilier de la tombe de Sennefer (TT 96B) avec le plancher de bois et les vitres protectrices. Phot. Pietri, Renaud, 2012. ( ) Renaud Pietri. 
Figure 12

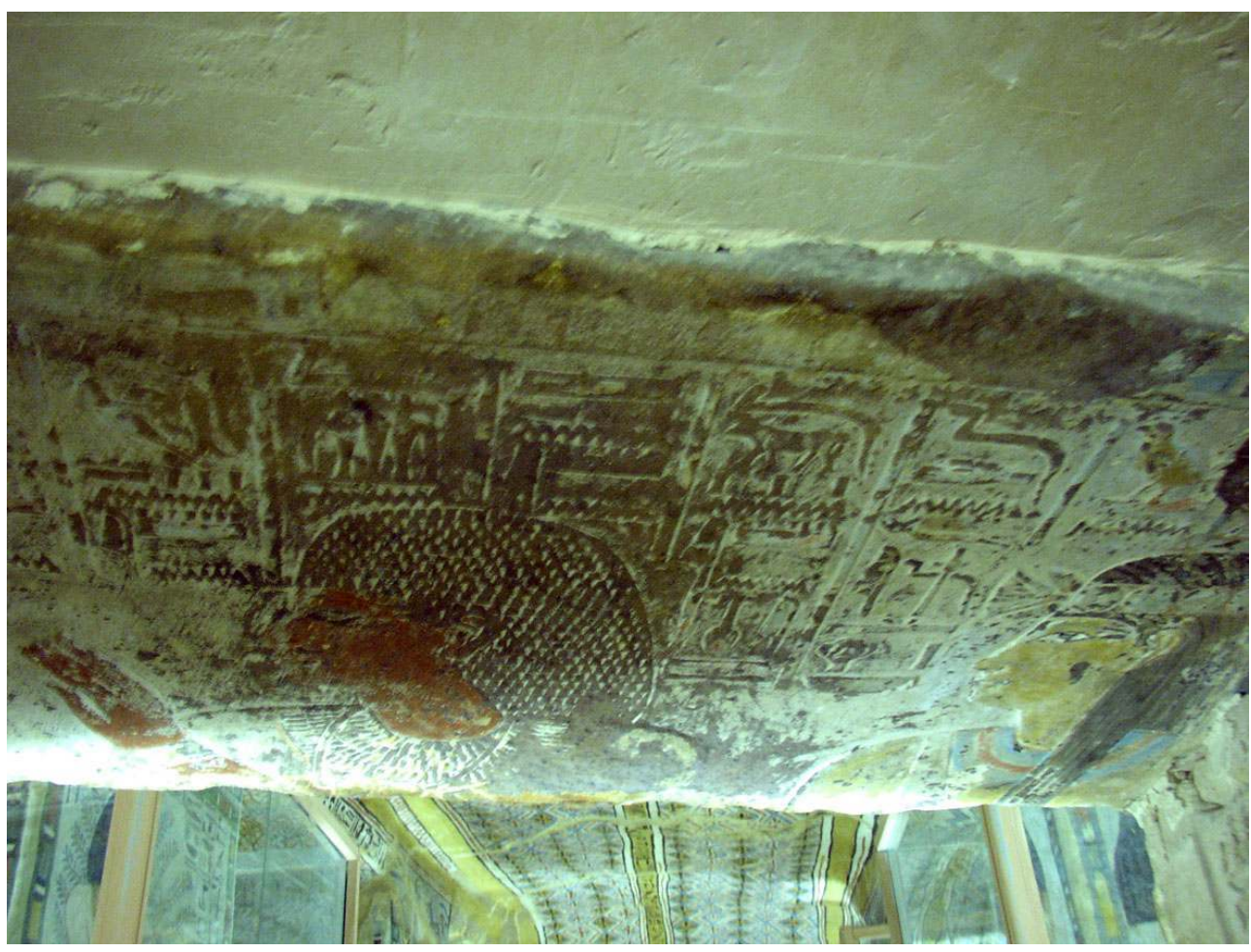

Plafond du passage entre l'antichambre et la chambre du caveau de la tombe de Sennefer (TT 96B).

Phot. Guilleux, Alain, 2007. ( ) Alain Guilleux.

12 Ces protections se révèlent être relativement efficaces étant donné que le caveau de Sennefer est peu visité par les groupes de touristes. L'isolement du monument et sa localisation hors des circuits touristiques habituels jouent en faveur de sa conservation. Les mesures de conservation préventive établies ont cependant une efficacité limitée. Outre l'aspect esthétique discutable de ces aménagements, le plancher rétrécit le caveau et les vitres, rarement nettoyées, gênent la vision des peintures murales. N'étant pas calibrées, elles ne protègent pas le haut des parois et la condensation s'y concentre.

Mettre en place un système de climatisation à l'instar de celui installé dans la salle du sarcophage de la Pyramide de Khéops ou de quelques tombes de la Vallée des Rois pourrait constituer une solution de conservation préventive. Ce type d'installation est cependant lourd et coûteux et ne convient pas à tout type de monument. Une étude est en cours afin de vérifier que l'air conditionné ne représente pas un risque supplémentaire de dégradations pour les peintures murales. La solution la plus efficace pour la conservation des peintures demeure la fermeture de la tombe. Cette décision soulève différentes questions éthiques et économiques, elle est donc rarement prise à moins d'une très grande urgence. La fermeture du caveau de Sennefer constitue donc une solution idéale mais difficilement envisageable. Il existe cependant des fac-similés - des copies fidèles du caveau original en trois dimensions et en grandeur réelle - qui pourraient tenir le rôle de substituts. Mais cette solution est-elle viable? Un remplacement éventuel est-il envisageable et serait-il au service de la conservation de la tombe réelle? 


\section{La réalisation des fac-similés de la tombe de Sennefer et leur rôle}

14 Le but premier de la fabrication du fac-similé reproduisant le caveau de la tombe de Sennefer, exécuté par la société Kodak-Pathé dans les années 1980, était de montrer le savoir-faire de la société en utilisant une technique unique. Une fois l'opération effectuée et le fac-similé montré dans quelques expositions temporaires, celui-ci et les photographies ayant servi à sa réalisation n'avaient plus de raison d'exister. L'idée originale était surtout de démontrer l'opérabilité du procédé Kodak avant même toute notion de conservation. Le choix s'est porté sur le caveau de Sennefer non pour les risques qu'il encourrait mais pour l'originalité de son décor et la bonne conservation de ses peintures. Le temporaire est finalement devenu pérenne, modifiant le rôle et le statut du fac-similé.

\section{La réalisation du fac-similé : une technique particulière}

15 Le fac-similé du caveau de Sennefer utilise une technique particulière rarement employée, excepté pour la reconstitution de la Salle des Taureaux de la grotte de Lascaux réalisée en 1980 et celle des fresques de Santorin en 1992 < lien http:// www.basictheatral.com/reconstitution_parietale.php>. La fabrication du premier facsimilé du caveau de Sennefer date de 1984-1985, l'idée ayant été émise au cours de l'été 1982. Si le procédé a été mis au point par la société Kodak-Pathé en 1980, le brevet a été racheté au début des années 1990 par la société lyonnaise Basic Théâtral.

La technique du fac-similé relief en matière synthétique avec transfert d'émulsion couleur se fonde sur le principe de la décalcomanie. À partir d'un tirage photographique, l'émulsion est détachée de son support pour la transférer sur des parois synthétiques irrégulières aux dimensions de la tombe réelle ${ }^{15}$. Tout a donc commencé par un travail dans la tombe originale de Sennefer où photographies des peintures et mesures du caveau ont été prises avec exactitude ${ }^{16}$, ni la chapelle, ni l'antichambre ne faisant l'objet de la reconstitution. $\mathrm{Au}$ total, ce sont vingt photographies des parois, vingt-deux des piliers, douze du plafond et une vingtaine de clichés de détail qui ont été réalisés, chacun en double exemplaire ${ }^{17}$. Afin de compléter les informations concernant le caveau et réaliser une réplique fidèle en trois dimensions, le topographe a pris les mesures précises du caveau ${ }^{18}$. Ce travail a permis d'obtenir une mémorisation globale du caveau de son état à l'époque. 
Figure 13

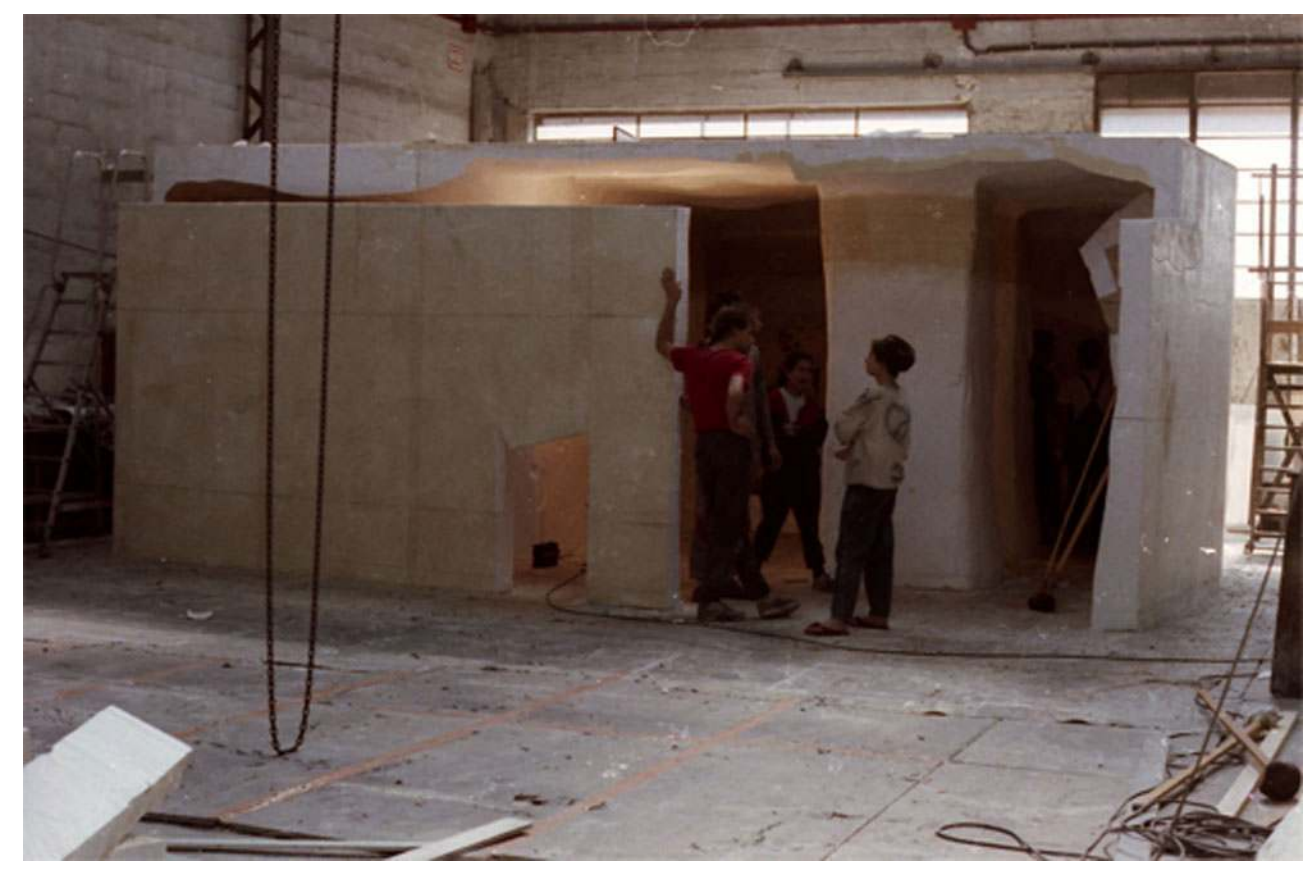

Support du fac-similé.

PHOT. BASIC THÉÂTRAL, 1985. ㄷ BASIC THÉÂTRAL.

17 Les données topographiques ont été exploitées par la société Basic Théâtral spécialisée dans la création de décors de théâtre et qui avait auparavant réalisé la reconstitution d'une partie de la grotte de Lascaux ${ }^{19}$. Cette société a fait sculpter les murs et piliers du caveau grandeur nature dans de grands panneaux de polystyrène expansé tandis que le plafond a été obtenu par l'assemblage de lames larges de $25 \mathrm{~cm}$ (fig. $\mathbf{n}^{\circ} \mathbf{1 3}$ ). Le découpage du fac-similé a l'avantage de le rendre léger, facilement transportable et rapidement montable. Ces fragments ont ensuite été affinés et poncés puis entoilés à l'envers et recouverts d'une résine à l'endroit afin de rendre le matériau résistant, de permettre le transfert de la gélatine photographique et de donner l'illusion des enduits originaux grâce à la granulation de la résine ${ }^{20}$. Une couche de peinture a été posée sur l'ensemble pour obtenir un fond chromatique neutre. Cette structure a ensuite reçu la gélatine détachée du support photographique. Chaque morceau a été placé avec exactitude sur le support polystyrène, collée par un côté. Sur l'autre côté, le papier-transfert a été mouillé afin qu'il se détache et que la gélatine reste collée sur la paroi ${ }^{21}\left(\right.$ fig. $\left.\mathbf{n}^{\circ} \mathbf{1 4}\right)$. 


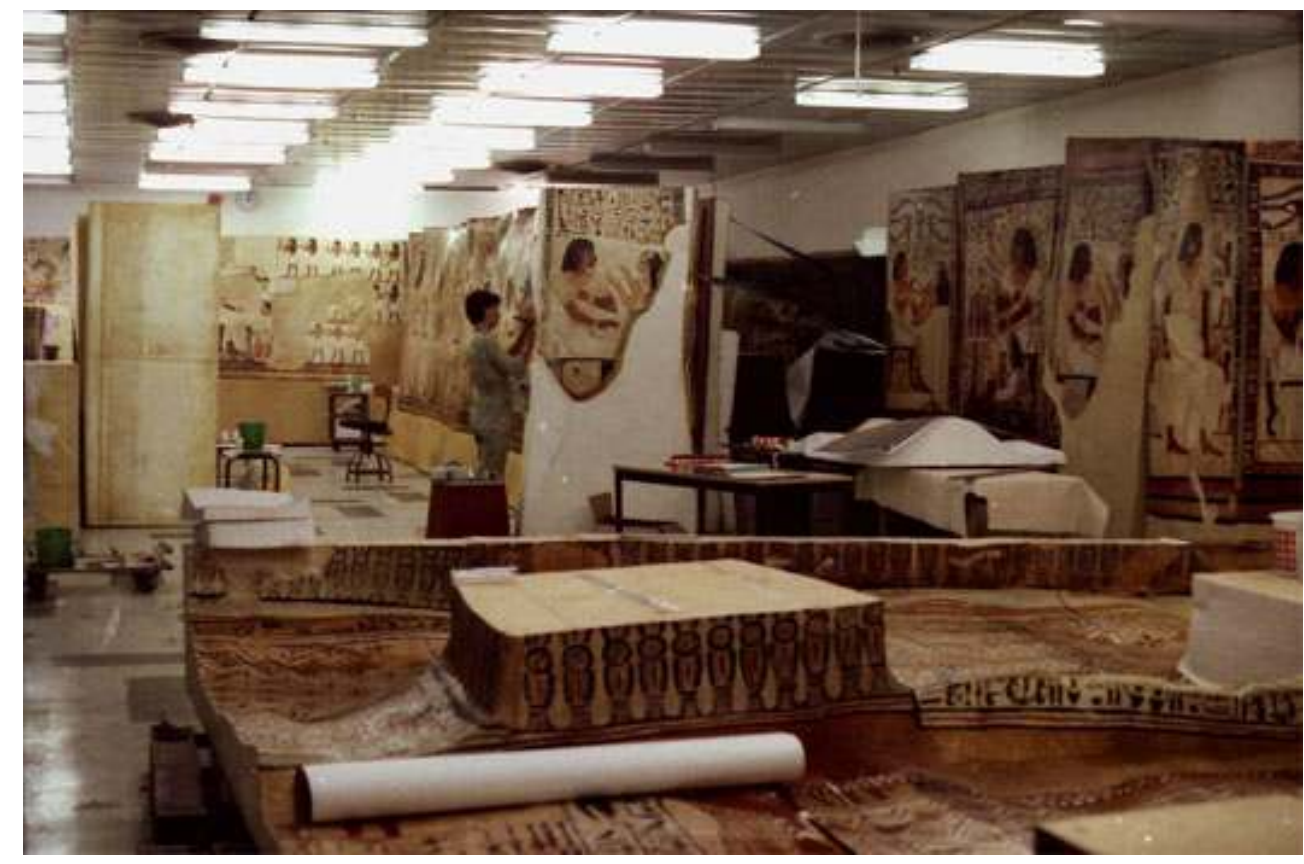

Vue des éléments démontés après pose de l'émulsion photographique.

Phot. Basic Théâtral, 1985. @ Basic Théâtral.

Une fois la tombe remontée et les joints cachés, le fac-similé est très proche de l'original, d'une grande fidélité "photographique». La technique du fac-similé du caveau de Sennefer a pour principal intérêt d'être très rigoureuse puisque la méthode de reproduction laisse peu de place à l'arbitraire humain et à l'interprétation artistique ${ }^{22}$. C'est une reconstitution scientifique pouvant faire l'objet d'études iconographiques. Il s'agit d'un «vrai » fac-similé dans le sens d'une copie parfaite de l'original même s'il utilise un matériau différent pour le support. Des matières synthétiques inertes ont d'ailleurs été choisies pour celui-ci afin de faciliter sa conservation; des matières naturelles auraient demandé trop de $\operatorname{soin}^{23}$. La duplication de ce fac-similé est aisée car les décalcomanies photographiques, supports de l'information visuelle, peuvent être tirées en de multiples exemplaires en fonction des besoins.

Ce procédé de création d'un fac-similé est cependant concurrencé par d'autres techniques, en particulier celle qui consiste à reproduire manuellement les décors à l'aide de colorants naturels sur des parois imitant le monument original, à l'aide de photographies projetées. Cette technique est la plus fréquemment employée pour la création de fac-similés de monuments, par exemple pour le fac-similé de la grotte de Lascaux $^{24}$ (reproduction de la Salle des Taureaux et du Diverticule Axial), celui de la tombe de Sennedjem ${ }^{25}$ et celui de la grotte d'Altamira ${ }^{26}$. Cette technique présente l'avantage de donner aux visiteurs une meilleure impression de découvrir un décor peint, de rendre « l'aura » du monument original. Une part de subjectivité demeure inhérente à l'emploi de cette technique; elle a cependant beaucoup évolué et s'exerce aujourd'hui dans des conditions scientifiques limitant la subjectivité de ceux qui reconstituent ${ }^{27}$.

Les nouvelles technologies permettent de créer des reconstitutions identiques à l'original, à l'instar des fac-similés récents de plusieurs tombes égyptiennes de la Vallée des Rois et de la Vallée des Reines ${ }^{28}$. Les données du relief et des décors de la tombe originale sont 
enregistrées avec un scanner ou un appareil 3D à main pour les recoins. Cet enregistrement est complété par un échantillonnage des couleurs prélevé manuellement et une série de photographies. Puis, ces données sont traitées par l'informatique en vue d'une reproduction exacte des décors et des reliefs sur différents supports. La pose du décor reprend le principe de la décalcomanie. À l'époque de sa conception, la technique du fac-similé de la tombe de Sennefer présentait donc des avantages indéniables pour l'élaboration de substituts analogiques. L'avancée en matière de nouvelles technologies a rendu quelque peu obsolète ce procédé. Les nouvelles techniques n'ont cependant pas encore fait leur preuve dans des monuments aussi exigus et aux parois aussi irrégulières que celui de Sennefer.

\section{Rôles et intérêt du substitut}

21 Dans la conjoncture actuelle, la notion de fac-similé souligne les contradictions des visiteurs. D'une part, la reproduction est au cœur de la modernité ; elle est à mettre en relation avec le développement de la diffusion de masse ${ }^{29}$. D'autre part, le culte de l'original et la recherche de l'authenticité restent vivaces, peut-être par volonté d'être confronté à l'objet unique, aujourd'hui si facilement reproductible. Le substitut sert à remplacer un objet que l'on n'a pas, soit parce qu'il est perdu, soit parce qu'on ne peut pas le déplacer, soit parce que l'on ne peut pas se le procurer ${ }^{30}$.

Le fac-similé du caveau de la tombe de Sennefer peut servir de substitut en cas de fermeture de la tombe réell ${ }^{31}$. Dans le projet d'origine de Kodak-Pathé, une fois montré en France dans diverses expositions temporaires, il aurait pu être offert à l'Égypte. Il aurait alors été présenté à proximité de l'original dans la perspective éventuelle de fermer la tombe de Sennefer au public. L'idée a cependant été abandonnée. La fermeture d'un monument demeure la solution idéale pour sa protection et le substitut peut alors servir "d'alibi ", de "légitimité ", de justification à la clôture du monument. Les reconstitutions doivent alors être présentées de préférence in situ et de façon clairement identifiable. La conformité du substitut, sa fidélité à l'original sont essentielles et le modèle reste la référence. L'existence d'une copie ne saurait entraîner la négligence puis l'abandon des originaux, ce qui serait contraire au but recherché32.

Le fac-similé de la tombe de Sennefer sert actuellement d'instrument didactique de diffusion culturelle. Il permet de mettre à portée du public un objet lointain. La valeur documentaire et esthétique du monument de référence est conservée dans la copie puisque la notion d'authenticité est sans portée artistique ${ }^{33}$. Supprimer les contraintes d'accès à la tombe réelle et les obligations de déplacement risque néanmoins de diminuer la prise de conscience du caractère exceptionnel de l'original et de son ancienneté.

La conception du fac-similé a également permis de faire une couverture photographique de l'intégralité du monument à un moment donné. La mémorisation et l'enregistrement de l'original sont des éléments essentiels dans une démarche de conservation préventive. La valeur mémorielle est encore plus forte si les objets de référence sont fortement dégradés ou détruits ${ }^{34}$. Dans cette perspective, les aquarelles et les relevés des Monuments Historiques réalisés au début du $\mathrm{XX}^{\mathrm{e}}$ siècle sont de précieux témoins. Des reproductions grandeur nature ont été fabriquées à partir de ces relevés, pour que le public ait une meilleure idée des monuments originaux ${ }^{35}$. De la même façon, en cas de disparition de la tombe de Sennefer, la couverture photographique et les fac-similés sont 
tout ce qu'il restera du caveau. Les substituts changent donc radicalement de signification s'ils ne sont plus couplés avec l'original ${ }^{36}$ mais leur valeur de témoin demeure essentielle.

\section{La présentation des fac-similés}

Deux fac-similés du caveau de la tombe de Sennefer ont été réalisés, l'un en 1985 et l'autre en 1987, respectivement actuellement en possession du musée de Tessé au Mans et du musée d'Hildesheim en Allemagne. En 1985, le premier fac-similé de la tombe de Sennefer est présenté à la Cité des Sciences de la Villette lors d'une exposition temporaire. Un an plus tard, la société Kodak-Pathé cherche un lieu pour déposer la reconstitution du caveau, de préférence un musée. En 1988, elle est donnée au Musée de Tessé du Mans. L'enjeu pour ce musée est alors de créer un nouvel espace susceptible d'accueillir le facsimilé, si possible en lien avec ses collections égyptiennes. Ce n'est qu'en 1998 qu'est formulée l'idée de la réalisation actuelle, à savoir la construction d'un cube en béton sur la façade arrière du bâtiment ancien, sous la terrasse côté jardin, en sous-sol. Cette réalisation a l'avantage d'être invisible de l'extérieur et de ne pas abîmer le paysage du parc de Tessé. L'ensemble communique avec le bâtiment principal permettant une entrée et un accueil communs et une certaine liberté d'aménagement intérieur. Les nouvelles salles créées remplissent ainsi leur mission de rassembler à la fois les collections égyptiennes du musée et les deux reconstitutions des tombes de Sennefer et de Nofretari. L'espace créé est de $546 \mathrm{~m}^{2}$ au total, dont $479,60 \mathrm{~m}^{2}$ de surfaces utiles. Les travaux pour la construction de ces salles ont débuté en octobre 2000 et se sont terminés un an après; l'inauguration a eu lieu le 22 novembre 2001. De 1988 à 2001, le fac-similé a été conservé démonté dans des réserves externalisées au Mans et n'a été montré que deux fois lors d'expositions temporaires, au Palais des Congrès pendant l'été 1992, puis, dans la salle Courboulay pendant l'été 1998.

La galerie égyptienne du musée de Tessé du Mans, étant en sous-sol, est accessible par un escalier suggérant la descenderie des tombes. L'intérêt de ces nouvelles salles est de pouvoir réunir objets antiques et reconstitutions de monuments, ce qui permet aux visiteurs d'apprécier plusieurs aspects du patrimoine égyptien. Selon le parcours standard, le visiteur passe d'abord par la reconstitution de la tombe de Nofretari sur deux niveaux, puis il remonte vers la tombe de Sennefer. La tombe de Nofretari est constituée de tirages photographiques collés sur des plaques d'aluminium planes. La technique employée pour la reconstitution de la tombe de Sennefer surprend et marque davantage le public. D'après leurs témoignages, cette dernière est celle que préfèrent généralement les visiteurs ${ }^{37}$. La reconstitution de la tombe de Sennefer s'est bien adaptée dans son espace muséal et est même devenue la pièce maitresse des collections égyptiennes (fig. $\mathbf{n}^{\circ}$ 15). 
Figure 15

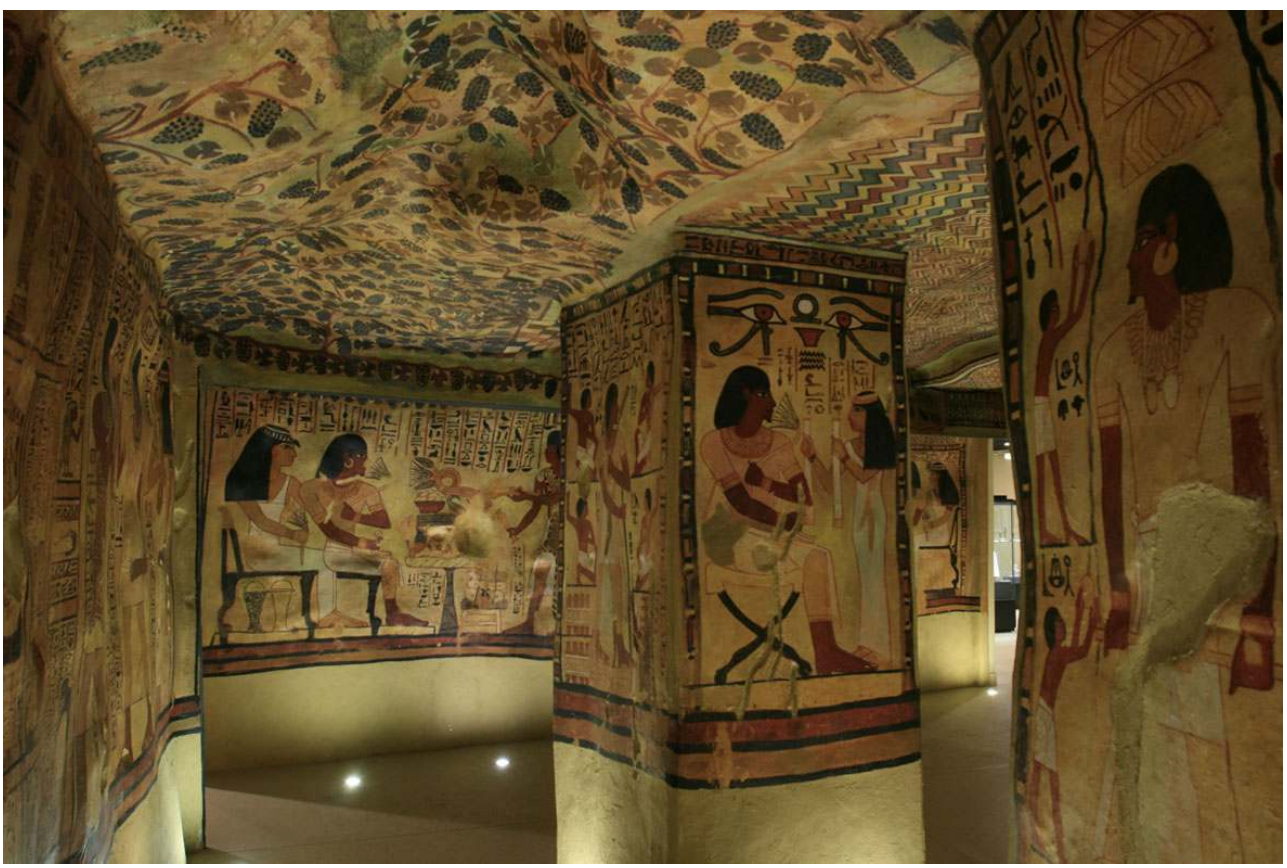

Vue générale du fac-similé au musée de Tessé du Mans dans sa présentation actuelle.

Phot. Poussin, Dominique, 2010. () Musée de Tessé, Le Mans.

Le second fac-similé du caveau de Sennefer, présenté aujourd'hui dans le musée Roemer et Pelizaeus d'Hildesheim, a été commandé et fabriqué en 1987 spécialement pour l'exposition temporaire "Ägyptens Aufstieg zur Weltmacht " $^{38}$. Il a ensuite été exposé dans divers musées, principalement en Allemagne, lors d'expositions temporaires, comme pour l'exposition « Ägypten: Götter, Gräber und die Kunst, 4000 Jahre Jenseitsglaube » qui s'est déroulée au Schlossmuseum de Linz (Autriche) ${ }^{39}$ en 1989. Le reste du temps, le fac-similé était démonté et mis en réserve. C'est seulement depuis huit ans qu'il est installé au musée Roemer et Pelizaeus de façon définitive (fig. $\mathbf{n}^{\circ} \mathbf{1 6}$ ). 
Figure 16

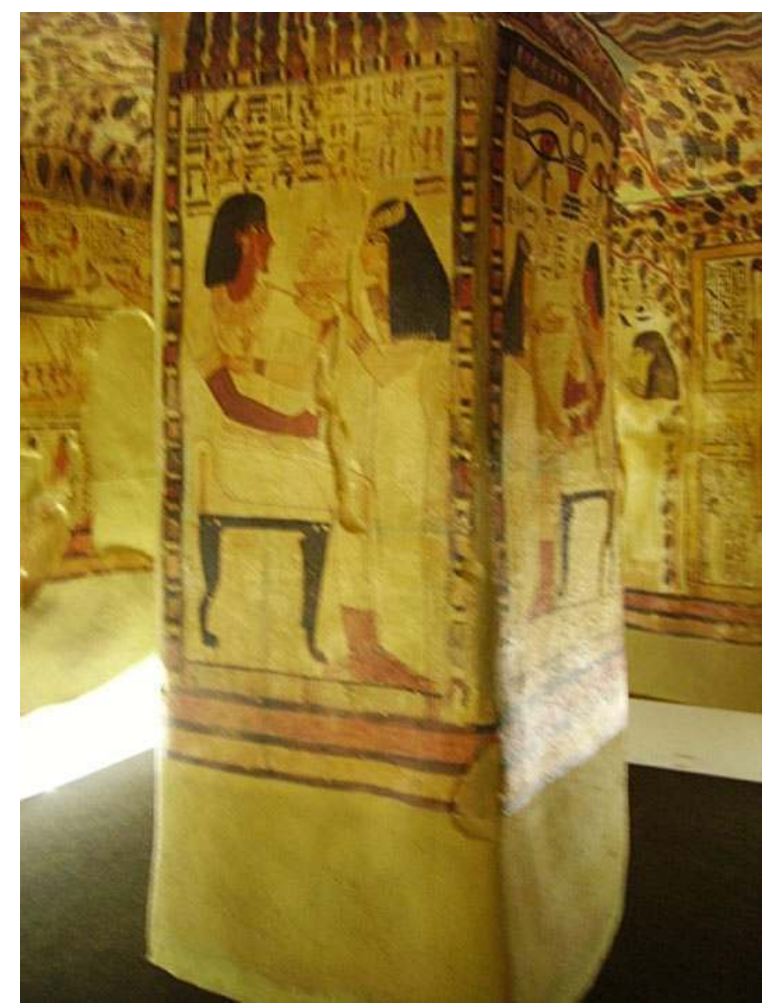

Vue générale du fac-similé au musée Roemer et Pelizaeus à Hildesheim dans sa présentation actuelle.

Phot. Maggio, Laëtitia, 2008. (c) Roemer- und Pelizaeus-Museum, Hildesheim.

La reconstitution de la tombe n'est pas exposée au sein de l'exposition permanente des antiquités égyptiennes, faute de place. Contrairement au musée du Mans où l'on a choisi de détacher et d'éloigner une paroi du caveau pour laisser passer les visiteurs, le facsimilé d'Hildesheim se rapproche de la tombe réelle de Sennefer dans laquelle l'accès se fait par un passage bas. Le visiteur doit donc se baisser voire s'agenouiller pour rentrer dans la chambre funéraire. L'entrée basse permet de se sentir complètement immergé dans le monument. Le fac-similé est ainsi utilisé dans le cadre d'activités pédagogiques proposées à des groupes d'enfants et d'élèves qui sont particulièrement impressionnés par ce mode de présentation. Celui-ci pose cependant le problème de l'accessibilité pour tous (fig. $\left.\mathbf{n}^{\circ} \mathbf{1 7}\right)$. 


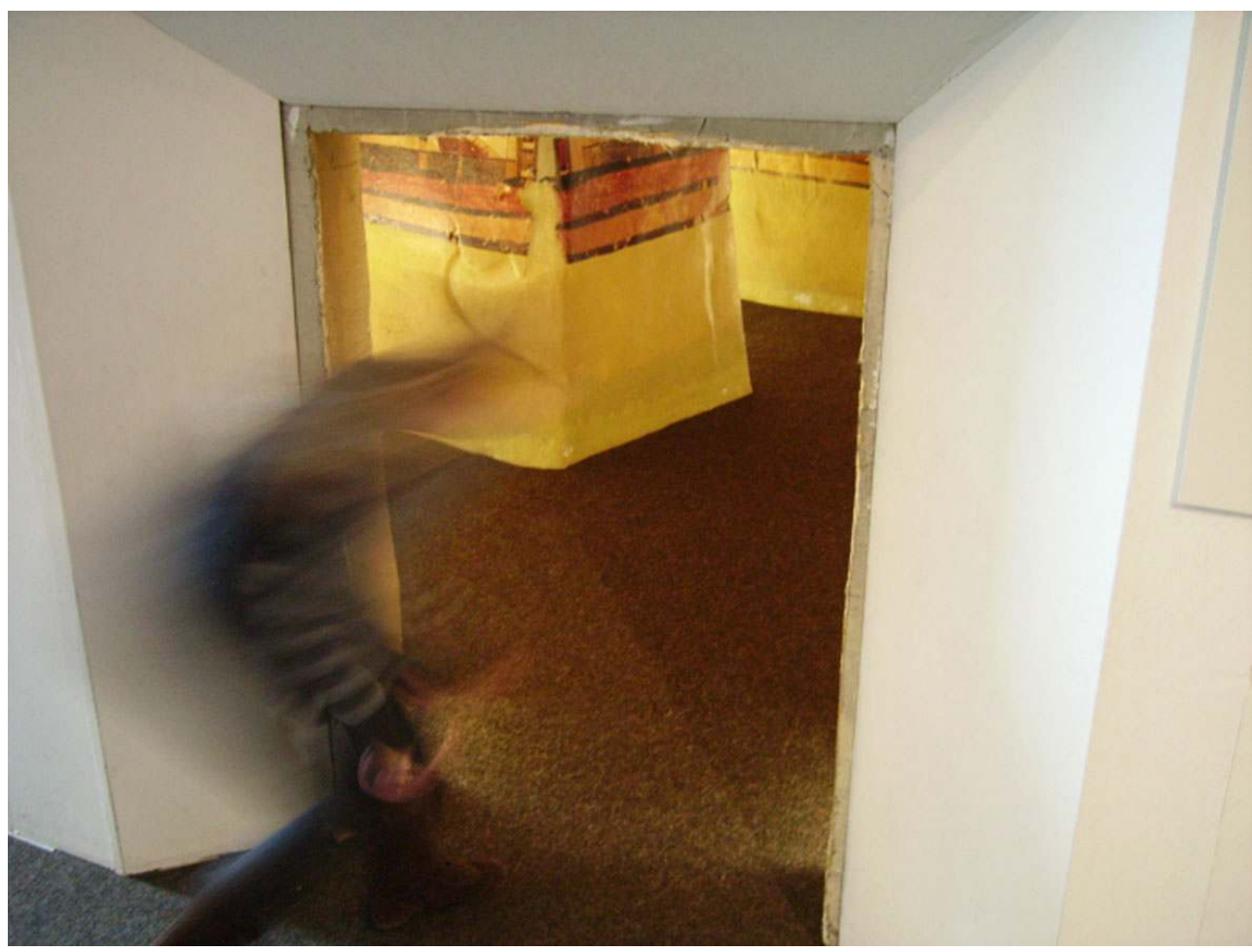

Entrée du fac-similé au musée Roemer et Pelizaeus à Hildesheim.

Phot. Maggio, Laëtitia, 2008. (c) Roemer- und Pelizaeus-Museum, Hildesheim.

\section{Le fac-similé entre permanent et fugitif : quelle légitimité pour sa conservation-restauration?}

A priori, la conservation préventive ne saurait s'attacher à ce qui est multipliable, ce qui peut être remplacé car «le caractère renouvelable suffit à en assurer la pérennité » ${ }^{40}$. La légitimité d'une démarche de conservation-restauration pour des fac-similés qui n'ont pas une «valeur culturelle originale » est sujette à caution. Dans le contexte patrimonial actuel, la situation est cependant complexe : des fac-similés non facilement multipliables, des originaux qui se dégradent ou disparaissent, des substituts témoignages d'une époque et d'une technique, sont des réalités à prendre en compte et qui poussent les différents acteurs à les conserver. À l'heure des choix économiques et de l'établissement de priorités pour la conservation des collections, quelle est la place de la conservation préventive pour un fac-similé ? Peut-on et doit-on les restaurer?

\section{Le fac-similé : un élément à conserver?}

Les fac-similés ont été conçus pour être éphémères puisque, en théorie, une fois dégradés, ils pouvaient être remplacés par un autre fac-similé complètement identique. L'objet authentique, lui, est unique et est donc le seul concerné par une démarche de conservation-restauration. La reproductibilité du fac-similé est exacte en théorie, mais la réalité est souvent plus complexe et il perd souvent son caractère multipliable, pour des raisons économiques ou matérielles. La technique employée pour la tombe de Sennefer 
porte en elle la capacité de reproduction à l'infini. Les photographies et les mesures topographiques effectuées dans la tombe réelle peuvent être réutilisées. La démarche pour réaliser les fac-similés reste la même offrant la possibilité d'avoir des reproductions identiques. Cependant, la production d'une copie de ce niveau de qualité a un coût élevé, ce qui incite à bien la conserver et à en limiter les altérations.

31 Le fac-similé peut devenir authentique, non comme l'original qu'il imite - qui a une valeur historique très forte due à son ancienneté - mais en tant que représentant d'une technique, d'un savoir-faire, d'un goût, témoins d'un contexte historique particulier. La première reconstitution d'un monument égyptien fut probablement celle de la tombe de Séthi I ${ }^{\text {er }}$ réalisée par Giovanni Battista Belzoni et peinte à la main ${ }^{41}$. Présentée à l'Egyptian Halls à Londres en 1821-1822, elle a rencontré un très grand succès et a marqué durablement les esprits. L'objet est entré dans les collections du Bristol City Museum en juillet 1900 et a reçu un numéro d'inventaire le 24 décembre $1929^{42}$. Ce type de monument n'est en général pas inscrit sur les inventaires des musées, ce qui les place en marge des collections permanentes. Le fac-similé de Belzoni est d'une grande valeur documentaire pour le rôle qu'il a joué dans l'histoire du goût et du spectacle. En outre, les relevés de l'équipe de Belzoni décrivent certains décors qui ont aujourd'hui disparu. Cela leur confère une très grande utilité en ce qui concerne la conservation et la restauration de la tombe réelle, même si cela n'était pas l'objectif de départ. Ces relevés sont dispersés dans différents musées; le fac-similé avait donc l'avantage de réunir tous ces dessins tout en offrant une expérimentation unique de l'espace. La reconstitution de Belzoni n'était cependant pas une copie fidèle de la tombe réelle mais en donnait une approximation, les défauts étant également intéressants dans une perspective historique. Tous les substituts qui ont été fabriqués dès la fin du XVIII siècle dans le cadre du Musée des Monuments Français ont également aujourd'hui la double valeur de témoignages historiques précieux et de sources documentaires essentielles. Ils montrent l'engouement pour la protection $\mathrm{du}$ patrimoine et sa diffusion tout en offrant une image du monument original, bien souvent dégradé ou même parfois disparu ${ }^{43}$. L'intérêt historique d'un fac-similé semble donc émerger à un moment ou à un autre de son histoire. Il devrait donc faire l'objet d'une conservation de qualité dans l'idée de devenir, un jour, un objet historique. Celui de la tombe de Sennefer s'inscrit d'autant mieux dans cette perspective qu'il est le témoin d'un mode de fabrication très particulier qui a marqué son temps.

\section{L'état de conservation des fac-similés de la tombe de Sennefer}

32 Les musées du Mans et d'Hildesheim tentent d'obtenir le meilleur état de conservation pour leur fac-similé, à travers des actions de restauration ou des démarches de conservation préventive. Malheureusement, le caractère instable des matériaux utilisés, les conditions de conservation longtemps fluctuantes et les nombreux déplacements pour des expositions temporaires ont contribué à leur vieillissement prématuré. Confronter les altérations relevées et l'historique de la conservation de chacun de ces fac-similés, à l'origine parfaitement identiques, permet de comprendre les facteurs de dégradation pour mieux les contrer.

33 La première altération remarquable est le jaunissement de la colle servant à fixer la couche photographique sur le support, par un phénomène photo-sensible. La lumière est donc un facteur de dégradation important pour les deux fac-similés qui ont surtout été détériorés lors de leur longue conservation en réserve ${ }^{44}$. Ils sont cependant encore soumis 
à la lumière du fait de leur présentation au public même si l'éclairage est minimal, réduit aux périodes d'ouverture, faible et protégé par des filtres. Le jaunissement est l'évolution naturelle de la colle d'origine. On peut difficilement lutter directement contre le jaunissement généralisé des fac-similés existants bien que la société Basic Théâtral propose actuellement un travail de retouches ponctuelles à l'aide de peintures aux couleurs étalonnées. L'alternative serait de refaire entièrement les fac-similés avec une technique améliorée et des matériaux plus stables dans le temps.

L'évolution des couleurs signifie-t-elle que le fac-similé n'est plus une reproduction valable, une "bonne reproduction" au sens d'Erwin Panofsky ${ }^{45}$ ? Elle n'est guère alarmante si l'évolution est uniforme, régulière et offre toujours aux spectateurs le même rapport de couleurs. Dans le monde symbolique de l'art égyptien où chaque couleur a une signification magique, certains rapports de couleurs sont essentiels et ne sauraient être dénaturés. Une étude colorimétrique a donc été envisagée. À l'aide d'un colorimètre électronique, des mesures de couleurs en système CIE L ${ }^{*}{ }^{*} b^{* 46}$ ont été effectuées pour une soixantaine de points des décors du caveau. Elles ont été prises aux mêmes endroits sur les photographies de la tombe réelle de Sennefer, sur le fac-similé du Mans et sur celui d'Hildesheim. En calculant les différences de couleurs entre deux zones d'un même facsimilé et en les comparant avec celles du monument original, l'uniformité du jaunissement peut être contrôlée. Les rapports de couleurs significatifs, comme la couleur de la peau des hommes et des femmes ou celle des objets cultuels, sont essentiels à préserver. Les mesures prises sur les deux fac-similés montrent que le jaunissement est uniforme excepté pour les blancs, notamment ceux des fonds, dont la couleur a varié de façon plus importante. Cette évolution est préoccupante car elle fausse l'impression globale de la tombe originale.

Comparer les couleurs du fac-similé du Mans et celui d'Hildesheim permet de vérifier si les différences de conditions de conservation ont influé sur l'évolution des couleurs. Après mesures, le fac-similé d'Hildesheim a globalement légèrement plus jauni que celui du Mans mais, dans le détail, cette évolution n'est pas la même pour toutes les scènes. L'éclairage actuel de ces deux reproductions est assez similaire, excepté pour les piliers. Il semble donc avoir peu d'impact sur le jaunissement des décors au contraire des conditions de conservation en réserve. On pourrait cependant préconiser la mise en place d'un éclairage se déclenchant au passage des visiteurs afin de limiter l'exposition à la lumière. 
Figure 18

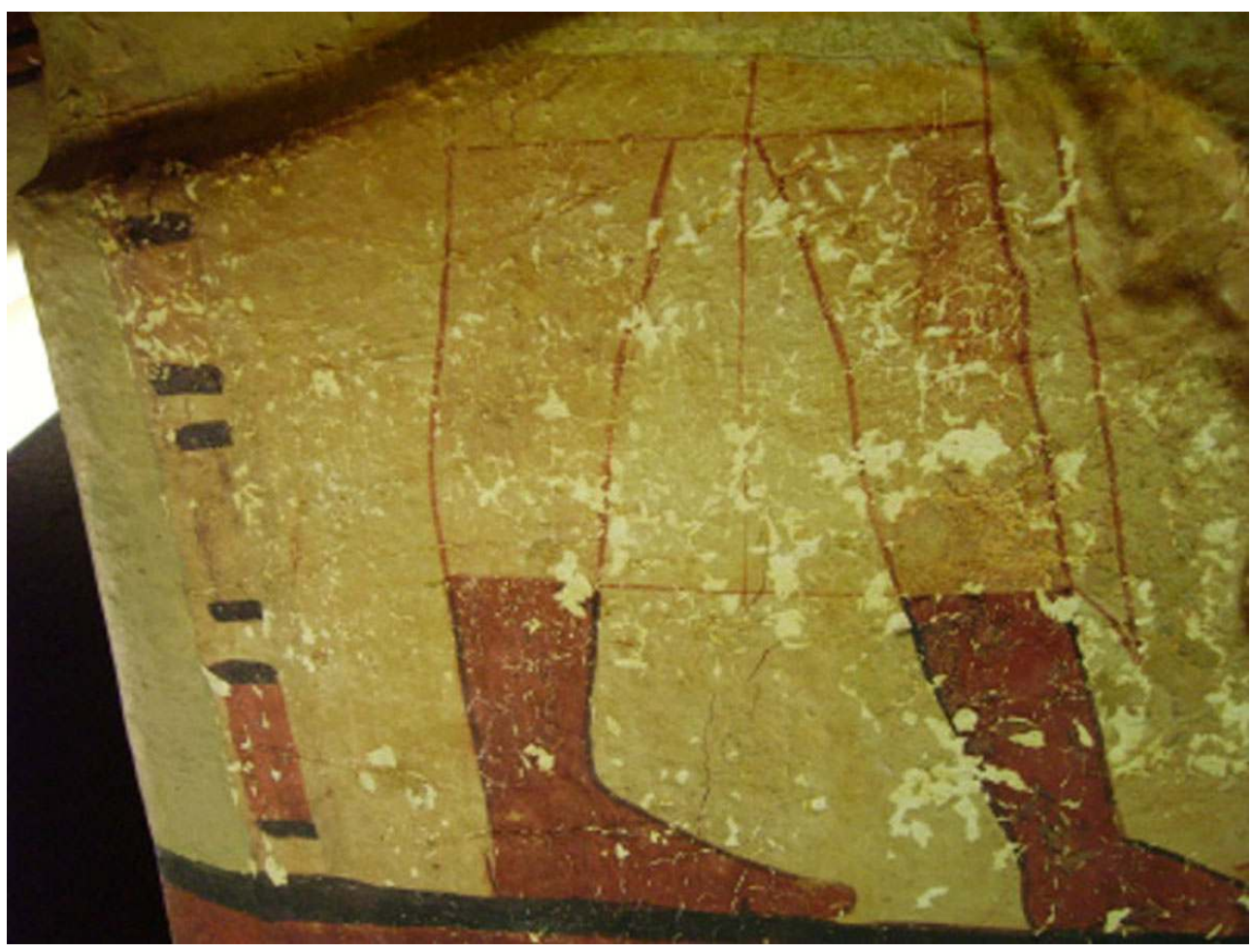

Couche de gélatine abîmée sur le fac-similé au musée Roemer et Pelizaeus à Hildesheim.

Phot. Maggio, Laëtitia, 2008. (c) Roemer- und Pelizaeus-Museum, Hildesheim.

Les conditions de température et d'hygrométrie sont également essentielles et sont à l'origine de quelques altérations. Une trop grande humidité favorise le développement de moisissures et une trop grande sécheresse celui de fissures sur la surface. Les dégradations observées sont aussi d'ordre mécanique, résultats de l'action des visiteurs. Le fac-similé placé au musée du Mans est marqué par quelques fines craquelures dues au vieillissement naturel de la gélatine, des plis dans la couche photographique qui sont des défauts de mise en œuvre et quelques taches résultant sans doute de l'évolution des pigments de la couche picturale. Le fac-similé d'Hildesheim présente d'importantes altérations de surface principalement dues aux conditions climatiques de conservation ${ }^{47}$. La couche de gélatine s'est détachée à certains endroits en s'enroulant sur elle-même laissant apparaitre le support clair (fig. $\mathbf{n}^{\circ} \mathbf{1 8}$ ). Des repeints assez grossiers gênent la lecture des décors reproduits (fig. $\mathbf{n}^{\circ} \mathbf{1 9}$ ). Avant 1998, date de la restauration du facsimilé, une fine couche de poussière recouvrait l'ensemble de la surface et adhérait à la couche de gélatine, des moisissures étaient également apparues sur l'un des piliers. 


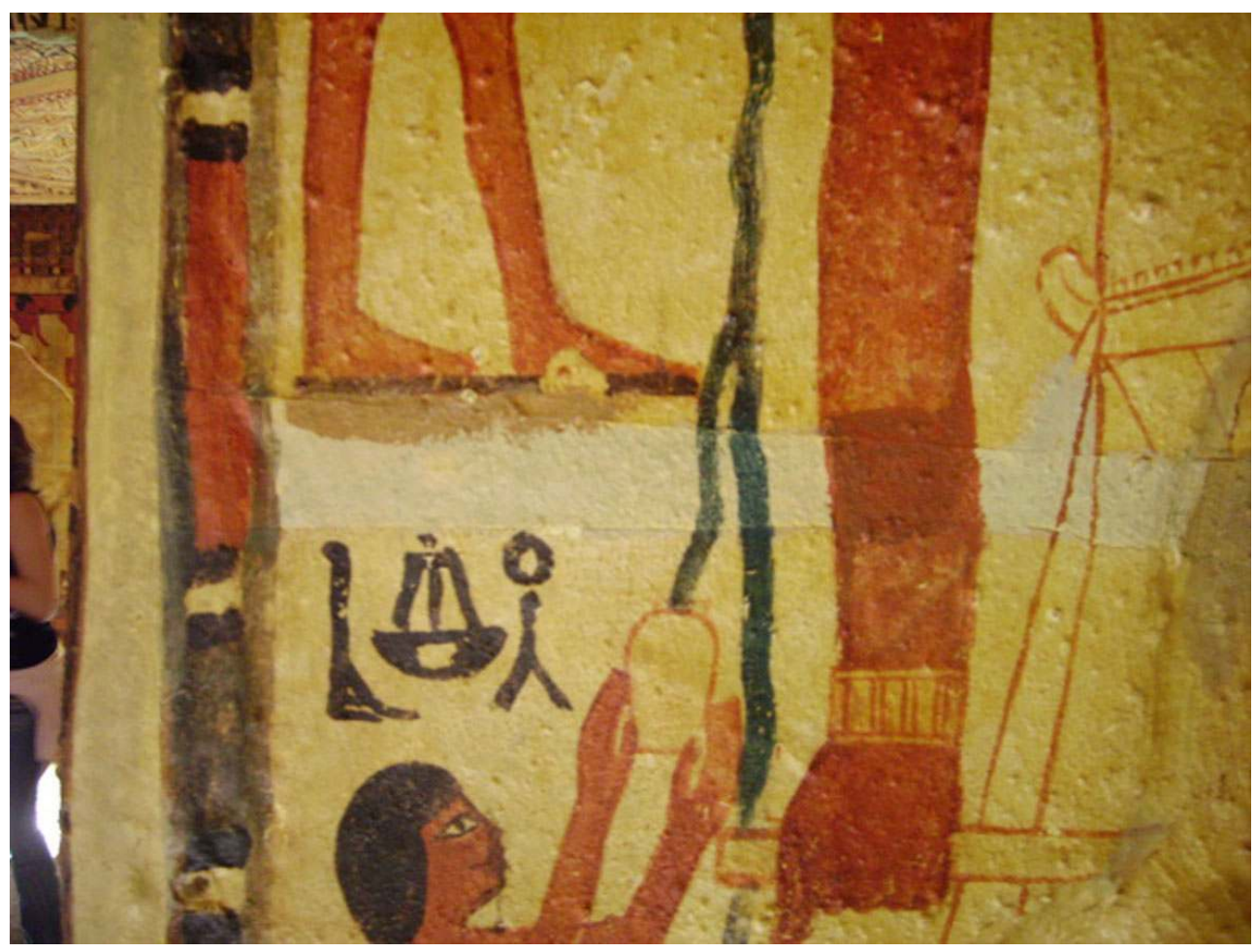

Repeints sur le fac-similé au musée Roemer et Pelizaeus à Hildesheim.

Phot. Maggio, Laëtitia, 2008. @ Roemer- und Pelizaeus-Museum, Hildesheim.

\section{Les différentes stratégies pour la conservation des fac-similés de la tombe de Sennefer}

Les musées du Mans et d'Hildesheim ont donc eu recours à des opérations ponctuelles de restauration afin de minimiser les dégradations. L'équipe de Basic Théâtral intervient régulièrement sur le fac-similé du Mans afin de l'entretenir. Des opérations ponctuelles sont ainsi effectuées pour recoller les craquelures de la couche photographique. Les griffures et enfoncements du support sont également comblés, puis éventuellement retouchés avec de la peinture. Les salles sont équipées d'un dispositif de contrôle du climat très performant. Un thermo-hygromètre est placé dans le monument. Les locaux sont climatisés et disposent d'un système de désenfumage.

Le Musée d'Hildesheim a opté pour une campagne de restauration de son fac-similé en $1998^{48}$. Les objectifs étaient la fixation de la couche de gélatine avec des colles, le nettoyage de la surface à l'aspirateur, à la brosse et à l'eau, le comblement de quelques zones détériorées avec un mélange à base de craie blanche, le retrait de certains repeints, la réintégration des lacunes à l'aide de pigments liés, la désinfection et le retrait des moisissures. Grâce à ces actions, l'état de conservation du fac-similé a pu être amélioré de façon significative même si par manque de temps toutes les zones n'ont pu être refixées et retouchées. Du fait de sa localisation dans l'ancien bâtiment du musée qui date des années 1970 et qui n'est pas équipé d'un système de climatisation, il est ardu de maintenir un environnement favorable à sa bonne conservation, l'air étant généralement trop sec. 
Dans une démarche de conservation préventive de la tombe originale de Sennefer par enregistrement de tous les décors, la bonne conservation des photographies originales est essentielle. Elles sont les sources primaires porteuses de l'information visuelle et peuvent servir à la réalisation de fac-similés à l'identique. Cependant, ces photographies ne peuvent elles-mêmes être prises de nouveau car elles montrent un état de la tombe qui n'existe plus. Elles offrent également une couverture totale du monument. Les conserver c'est toutefois aller à l'encontre de leur objectif originel. Les photographies sont des matériaux fragiles dont le vieillissement est encore mal connu. La poussière, l'acidité des matériaux en contact, les variations brusques des conditions climatiques sont autant de facteurs d'altération pour les photographies ${ }^{49}$. Ce sont souvent les matériaux constitutifs eux-mêmes qui se dégradent. Pour le caveau de Sennefer, les photographies originales, formées de deux lots identiques, sont conservées dans les bureaux du département des Antiquités égyptiennes du musée du Louvre. Elles sont en très bon état de conservation dans un lieu qui a l'avantage de bénéficier d'un environnement stable et d'être peu fréquenté. Elles sont à l'abri de la lumière et ne sont pratiquement jamais manipulées. L'idéal serait de numériser ces photographies pour avoir une garantie de leur conservation. Cependant, pour que cette numérisation soit efficace, elle doit être d'une grande qualité, ce qui nécessite un équipement adéquat et des fonds (fig. $\mathbf{n}^{\circ} \mathbf{2 0}$ ). Les photographies de la face d'un pilier - «l'offrande du lotus » - ont également été assemblées numériquement lors d'un essai d'archéosynthèse ${ }^{\circledast}$ du décor du caveau ${ }^{50}$.

Figure 20

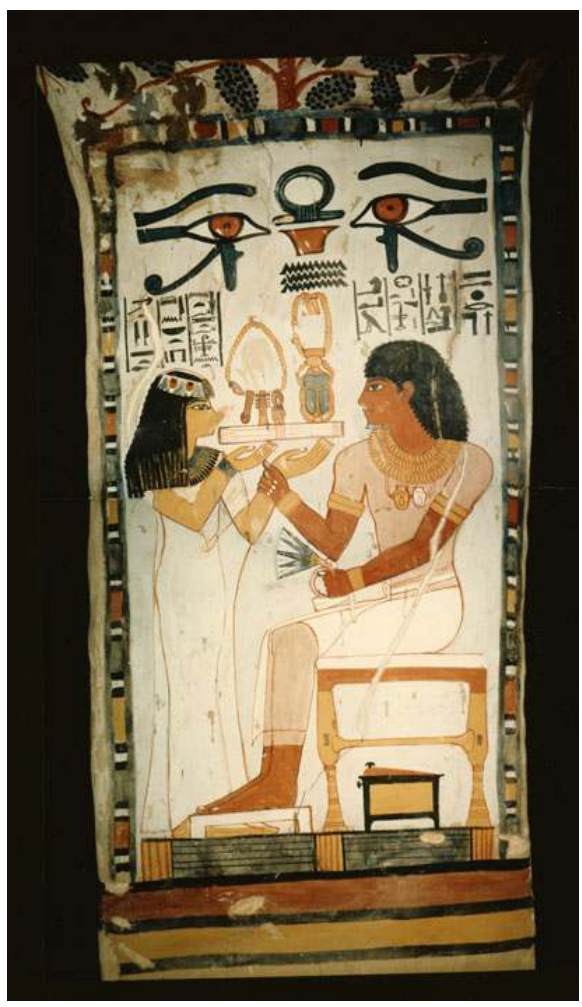

Scène numérisée à partir des photographies originales. Phot. Bovot, Jean-Luc, 1984. (c) Fondation Kodak Pathé. 


\section{Conclusion}

40 danger. Une démarche de conservation préventive serait une attitude idéale pour cette
tombe afin de pouvoir prolonger l'existence de ses décors peints exceptionnels. Cependant, mettre la conservation préventive au service d'un monument égyptien est complexe et cette solution devient, dans ce cas, souvent peu vraisemblable. Dans ce contexte, la fermeture de la tombe est envisageable ainsi que son remplacement par son fac-similé. Toutefois, même si les reproductions ont un succès considérable au sein de l'institution muséale, cela est moins sûr in situ.

41 Le public est fortement attiré par l'authenticité, la valeur historique d'un monument aussi ancien qu'un tombeau égyptien étant essentielle pour la juste appréciation du monument. Accepter de visiter un fac-similé en lieu et place de l'original ne se produit que dans certains cas très précis, en l'occurrence, si le monument original n'est pas disponible de par sa localisation ou de par son état, et si le public est bien informé des choix qui lui sont imposés. Le fac-similé est un «monument-document» dont la légitimité se fonde sur le fait qu'il est conforme à une image du monument original. Une reconstitution abîmée, en particulier si certains décors ne sont plus lisibles, ne remplit plus son rôle de substitut.

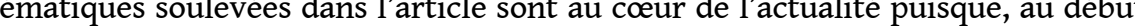
de l'année 2011, l'Égypte a annoncé la fermeture de la tombe de Toutânkhamon, la plus célèbre de la Vallée des Rois, pour la remplacer par un fac-similé. Du reste, les tombes les plus en danger des nécropoles royales thébaines semblent être promises au même sort. Celles de Néfertari et de Séthi I ${ }^{\mathrm{er}}$ déjà fermées aux visites depuis de nombreuses années, et celle de Thoutmosis III, souffrant de l'afflux touristique, vont être remplacées par des reconstitutions réalisées par la société Factum Arte. La technique utilisée repose sur le traitement informatique des données. Effectivement, les possibilités techniques autorisées par le développement des nouvelles imageries sont bien plus grandes aujourd'hui qu'elles ne l'étaient à l'époque de la création de la copie de la tombe de Sennefer. Ces évolutions laissent espérer une utilisation croissante du fac-similé comme substitut de l'original dans une volonté de le préserver tout en portant sa connaissance au plus grand nombre. Concilier tourisme de masse et préservation du patrimoine est dès lors envisageable.

\section{NOTES}

1. - Voir l'analyse scientifique des peintures de la chapelle, effectuée en 2002 par une équipe du Laboratoire de Recherche des Monuments Historiques. HUGON, Paulette, AUSSILLOUX, Aude. ÉGYPTE. Cheikh Abd-El-Gournah, Tombe de Sennefer (TT 96), chapelle funéraire. Peinture murale (XVIIIe dynastie, XVe siècle avant J.-C.) : étude stratigraphique et analyses physico-chimiques. Champs-surMarne : LRMH, janvier 2002, p. 14-17. 
2. - TAVIER, Hugues. «Materiam Superabat opus : la conservation-restauration de la chapelle de Sennefer ». Égypte, Afrique et Orient, mars $2007, \mathrm{n}^{\circ} 45, \mathrm{p} .38$.

3. - Le caveau a été fréquemment étudié et publié dès sa découverte. Voir, en particulier, les dessins de Robert Hay de Linplum en 1826, Philippe Virey en 1898 et Nina et Norman de Garis Davies dans les années 1920. Quant à la chapelle, elle n'a fait l'objet que de peu de commentaires excepté l'article de Robert Mond en 1902-1903 qui l'a vidée et effectué des nettoyages sévères. MOND, Robert. « Report on work done in the Gebel esh Sheikh, Abd el-Kurneh at Thebes, january to march 1903 ». ASAE Annales du Service des Antiquités égyptiennes, 1904, V, p. 100-101. Depuis 1999, la Mission archéologique dans la Nécropole thébaine (MANT) dépendante de l'Université de Bruxelles assure la conservation-restauration et l'étude des peintures murales de la chapelle.

4. - Les altérations des peintures de la chapelle ont été étudiées dans le mémoire de M.S.T. Conservation-restauration d'Aude Aussilloux. AUSSILLOUX, Aude. La conservation des peintures murales de la chapelle de Sennefer, Cheikh Abd-el-Gournah, Égypte. Mémoire de M.S.T. Conservationrestauration. Paris : Université de Paris I, Panthéon-Sorbonne, 2000-2001, non publié.

5. - TAVIER, Hugues. « Materiam Superabat opus : la conservation-restauration de la chapelle de Sennefer ». Égypte, Afrique et Orient, mars 2007, n45, p. 39.

6. - Voir les résultats dans MORA, Paolo, MORA, Laura, PORTA, Eduardo. "Conservation et restauration de la tombe de Néfertari dans la Vallée des Reines ». Preprints, Comité de l'ICOM pour la conservation. 9th Triennial Meeting, Dresden, German, Democratic Republic. 26-31 August 1990. Los Angeles : ICOM, 1990, p. 521. La tombe de Nefertari fait $250 \mathrm{~m}^{3}$ et, en temps normal, les conditions climatiques y sont régulières $-30^{\circ} \mathrm{C}$ pour la température et $30 \%$ d'humidité relative - avec peu de variations. Dans cette pièce au volume réduit, les moindres apports extérieurs de chaleur et de vapeur d'eau modifient rapidement et durablement les conditions climatiques. Les auteurs de l'étude notent que «la présence de quatre personnes pendant une heure dans la chambre funéraire fait augmenter l'humidité relative de plus de dix points et en même temps la température augmente d'un degré et demi ». Revenir aux conditions climatiques « normales » est un procédé long : plus de deux jours pour la tombe de Néfertari.

7. - LE FUR, Daniel. La conservation des peintures murales des temples de Karnak. Paris : Recherche sur les Civilisations, 1994, p. 139.

8. - COLLECTIF. Reconstitution du caveau de Sennefer dit "Tombe aux vignes »: Thèbes-Ouest, Cheikh Abd el Gournah, XVIII dynastie. Paris : Fondation Kodak Pathé, 1985, p. 21.

9. - Notamment son utilisation comme magasin d'antiquités pendant toute la fin du XIX $\mathrm{X}^{\mathrm{e}}$ siècle et le début du XX ${ }^{\mathrm{e}}$ siècle mais aussi, auparavant comme étable. Concernant l'histoire des utilisations de la chapelle, voir TEFNIN, Roland, PÉRIER-D'IETEREN, Catheline. « Archéologie et conservationrestauration dans les chapelles de Sennefer (TT96) et Aménémopé (TT29), à Cheikh abd-elGournah ». BSFE Bulletin de la Société Française d'Égyptologie, 2002, n¹54, p. 21 et AUSSILLOUX, Aude. La conservation des peintures murales de la chapelle de Sennefer, Cheikh Abd-el-Gournah, Égypte. Mémoire de M.S.T. Conservation-restauration. Paris: Université de Paris I, PanthéonSorbonne, 2000-2001, non publié.

10. - COLLECTIF. Reconstitution du caveau de Sennefer dit "Tombe aux vignes ": Thèbes-Ouest, Cheikh Abd el Gournah, XVIII dynastie. Paris : Fondation Kodak Pathé, 1985, p. 14.

11. - VIREY, Philippe. «La tombe des vignes à Thèbes ». RT Recueil de travaux relatifs à la philologie et à l'archéologie égyptiennes, $1898, \mathrm{n}^{\circ} 20, \mathrm{p} .211-223$. Bien que les relevés de Virey ne soient pas scientifiquement exacts, ils montrent bien cependant qu'entre 1898 et 1985 des fragments peints ont disparu.

12. - Les campagnes de conservation-restauration de la chapelle sont menées par la Mission Archéologique de la Nécropole Thébaine (ULB) depuis janvier 2001, sous la direction de Roland Tefnin puis de Laurent Bavay, à raison d'une campagne par an.

13. - COLLECTIF. Reconstitution du caveau de Sennefer dit «Tombe aux vignes »: Thèbes-Ouest, Cheikh Abd el Gournah, XVIII dynastie. Paris : Fondation Kodak Pathé, 1985, p. 21. 
14. - Les peintures murales sont menacées par le contact direct des visiteurs avec les parois (frottement des sacs des visiteurs, mains sur les peintures, etc.). Le passage bas entre l'antichambre et la chambre du caveau est particulièrement abîmé à cause des frottements réguliers à cet emplacement. Les dégradations sont d'ordre mécanique mais aussi chimique à cause de l'acidité de la transpiration des visiteurs.

15. - KURZ, Marcel. «Histoire d'une reconstitution ». Dans COLLECTIF. Reconstitution du caveau de Sennefer dit "Tombe aux vignes": Thèbes-Ouest, Cheikh Abd el Gournah, XVIII dynastie. Paris: Fondation Kodak Pathé, 1985, p. 20.

16. - Le projet de reconstitution a été effectué par Monique Nelson, Marcel Kurz et Jean-Luc Bovot, en collaboration avec une mission archéologique égyptienne dirigée par Dr Fathy Hassanein. EGGEBRECHT, Arne et alii. Ägypten: Götter, Gräber und die Kunst, 4000 Jahre Jenseitsglaube. Band II : Das Grab des Sennefer. [Katalog zur Austellung : Schlossmuseum Linz 9. April Bis 28. September, 1989]. Linz : Oberösterreichisches Landesmuseum, 1989, p. 77.

17. - Les techniques photographiques sont décrites dans BOVOT, Jean-Luc. « La Reconstitution de la tombe de Sennefer ». Dans TEFNIN, Roland (éd.). La peinture égyptienne ancienne, un monde de signes à préserver. Monumenta aegyptiaca, 7. [Actes du colloque international, Bruxelles, 1994]. Bruxelles : Fondation égyptologique Reine Élisabeth, 1997, p. 106.

18. - Pour les détails techniques, voir KURZ, Marcel. «Histoire d'une reconstitution ». Dans COLLECTIF. Reconstitution du caveau de Sennefer dit "Tombe aux vignes »: Thèbes-Ouest, Cheikh Abd el Gournah, XVIII dynastie. Paris : Fondation Kodak Pathé, 1985, p. 21.

19. - CUNILLERA, Alain, BRIEZ, Serge. «Les fac-similés reliefs en matières synthétiques avec transfert d'émulsion couleur». Dans COLLECTIF. 50 ans après la découverte de Lascaux. [Journées internationales d'étude sur la conservation de l'art rupestre, Périgord, 20-23 août 1990]. Périgueux : ICOM, 1990, p. 165.

20. - BOVOT, Jean-Luc. « La Reconstitution de la tombe de Sennefer ». Dans TEFNIN, Roland (éd.). La peinture égyptienne ancienne, un monde de signes à préserver. Monumenta aegyptiaca, 7. [Actes du colloque international, Bruxelles, 1994]. Bruxelles: Fondation égyptologique Reine Élisabeth, 1997, p. 108.

21. - Pour les détails techniques, voir CUNILLERA, Alain, BRIEZ, Serge. « Les fac-similés reliefs en matières synthétiques avec transfert d'émulsion couleur». Dans COLLECTIF. 50 ans après la découverte de Lascaux. [Journées internationales d'étude sur la conservation de l'art rupestre, Périgord, 20-23 août 1990]. Périgueux: ICOM, 1990, p. 165. KURZ, Marcel. "Histoire d'une reconstitution ». Dans COLLECTIF. Reconstitution du caveau de Sennefer dit "Tombe aux vignes": Thèbes-Ouest, Cheikh Abd el Gournah, XVIII dynastie. Paris : Fondation Kodak Pathé, 1985, p. 24.

22. - Associant l'exactitude mécanique de la photographie et une construction grandeur nature, le fac-similé relief en matière synthétique avec transfert d'émulsion couleur tel qu'il a été utilisé pour le caveau de Sennefer semble correspondre à la définition de la "bonne reproduction » formulée par différents théoriciens. Voir BENJAMIN, Walter. «L'œuvre d'art au temps de sa reproductibilité mécanique (1936)». Dans Sur l'art et la photographie. Paris : Éditions Carré, 1997, p. 21. Walter Benjamin différencie la reproduction manuelle et celle technique. Ainsi, «la reproduction technique (...) laisse intacte la consistance de l'œuvre d'art " même si elle ne remplacera jamais son authenticité. Pour Erwin Panofsky, ce n'est pas la reproduction qui falsifie l'original mais « la falsification du processus de reproduction par l'intervention humaine ». Pour qu'elle soit efficace, il faut qu'il s'agisse d'une «bonne reproduction » qui transmette non pas l'illusion de l'original mais l'intention de l'œuvre d'art; cette «bonne reproduction » ne peut être réalisée que dans la sphère du "mécanico-anorganique " pour rendre infime la frontière entre original et copie. Voir PANOFSKY, Erwin. « Original et reproduction en fac-similé (Original und Faksimilereproduktion, 1930) ». Les cahiers du Musée National d'art moderne, 1995, n53, p. 48. De même, Aloïs Riegl, en prévoyant l'amélioration technique des reproductions, notamment par une combinaison de la photographie avec la reproduction en fac-similé, les envisageait comme 
des substituts valables d'originaux satisfaisant à la fois les demandes de «valeur historique » et de « valeur d'ancienneté ». RIEGL, Aloïs (trad. D. Wieczorek). Le culte moderne des monuments. Son essence et sa genèse (1903). Paris : Seuil, 1984, p. 84-85.

23. - CUNILLERA, Alain, BRIEZ, Serge. «Les fac-similés reliefs en matières synthétiques avec transfert d'émulsion couleur ». Dans COLLECTIF. 50 ans après la découverte de Lascaux. [Journées internationales d'étude sur la conservation de l'art rupestre, Périgord, 20-23 août 1990]. Périgueux : ICOM, 1990, p. 166.

24. - Le fac-similé Lascaux II ouvre ses portes le 18 juillet 1983. <voir lien http:// www.lascaux.culture.fr/\#/fr/chrono.xml>.

25. - Ce fac-similé a été réalisé par une équipe du musée du Louvre en 1999. Cette réalisation a nécessité une couverture photographique de la tombe, la prise de mesures topographiques et les décors ont été peints en respectant l'échantillonnage des couleurs. Voir ZIEGLER, Christiane, BOVOT, Jean-Luc. A tumba de Sennedjem em Deir El-Medina [exposition Sao Paulo, Museu de Arte Brasileira-MAB, 2 de maio a 22 de julho de 2001]. Sao Paulo: Fundacao Armando Alvares Penteado, 2001.

26. - La «neocueva » est la reproduction de la grotte d'Altamira dans son état d'origine; le support et les pigments utilisés sont à dessein d'une composition proche de celle des matériaux du Paléolithique. Voir LASHERAS CORRUCHAGA, José Antonio, DE LAS HERAS, Carmen. « Un facsimilé, le choix pour Altamira ». In Situ, revue des patrimoines [en ligne], 2010, n 13 [consulté le 02/01/2012]. < lien hoir http://www.insitu.culture.fr/article.xsp? numero=13\&id_article=lasheras3D-380 >.

27. - L'exactitude n'est cependant pas toujours un but en soi lorsqu'on produit un fac-similé. Pour reprendre les termes de Bernard Deloche, le substitut analytique ne reproduit pas un monument spécifique avec exactitude mais est simplement un support pour des animations pédagogiques tandis que le substitut analogique «fournit une simulation d'effets et privilégie l'apparence sensible ». DELOCHE, Bernard. "Les substituts dans les musées ». ICOM, Lettre du Comité National français, mai 1999, $\mathrm{n}^{\circ} 25$, p. 11. Pour des exemples, voir GROGNET, Fabrice. «Mémoires de pierre... et de papier!». La lettre de l'OCIM, 2003, n 89, p. 15. PHILIPPE, Michel, SERMET, Christian. « La grotte du muséum de Lyon ». La Lettre de l'OCIM, juillet-août 2002, n 82, p. 4.

28. - Les fac-similés de la tombe de Séthi ${ }^{\mathrm{er}}$, de celle de Thoutmosis III et de celle de Toutankhamon ont respectivement été créés en 2002, 2003 et 2010 par la société Factum Arte et son initiateur Adam Lowe. Voir COLLECTIF. Immortal pharaoh : the Tomb of Thutmose III. [exposition, Edimbourg, City Art Centre, 1st October 2005 - 8 January 2006]. Madrid ; Copenhague : Factum Arte; United Exhibits Group, 2005, p. 177-184, et CHARTIER-RAYMOND, Maryvonne, CRANÇON, Sophie. "Dans la tombe de pharaon: le fac-similé de la chambre funéraire de Thoutmosis III ». Archeologia, novembre 2006, n438, p.39. <voir lien http://www.factumarte.com/ind/2/Conservation>.

29. - Par exemple, l'exposition itinérante « Toutânkhamon : son tombeau et ses trésors » qui a été présentée dans une dizaine de villes européennes depuis 2008 et qui montre plus de mille objets, répliques grandeur nature du mobilier funéraire de Toutânkhamon, a eu un succès considérable.

30. - Pour cette idée, voir DELOCHE, Bernard. Le musée virtuel : vers une éthique des nouvelles images. Paris : PUF, 2001, p. 185. DELOCHE, Bernard. «Et si le substitut valait plus que l'original ». Dans GUILLEMARD, Denis (dir.). Du moulage au fac-similé, diffusion du patrimoine et conservation préventive. [Ve Journées-débats organisées par le DESS de conservation préventive de l'Université de Paris I, 10 et 11 mai 2001]. Paris : ARAAFU, avril 2002, p. 17.

31. - Le projet du Conseil Suprême des Antiquités égyptiennes de remplacer la visite de quatre tombes qui sont actuellement fermées ou qui le seront prochainement - la tombe de Thoutmosis III, de Toutankhamon, de Séthi $\mathrm{I}^{\mathrm{er}}$ et de Nefertari - par la visite de leur fac-similé remplit cette fonction. LEBLANC, Christian, LECUYOT, Guy, PIMPAUD, Alban-Brice. « Un projet de centre de 
recherche, d'exposition et de documentation pour le site archéologique de Thèbes-Ouest ". Cahiers Supplémentaires des Memnonia, 2010, n², p. 271-281.

32. - GUILLEMARD, Denis. "Fidélité et trahison : le simulacre du substitut ». Dans GUILLEMARD, Denis (dir.). Du moulage au fac-similé, diffusion du patrimoine et conservation préventive. [Ve Journéesdébats organisées par le DESS de conservation préventive de l'Université de Paris I, 10 et 11 mai 2001]. Paris : ARAAFU, avril 2002, p. 13.

33. - C'est une notion purement historique. Voir GILSON, Étienne. Peinture et réalité. Paris : Librairie philosophique J. Vrin, 1972, $2^{\mathrm{e}}$ édition, p. 77.

34. - GUILLEMARD, Denis. «Fidélité et trahison : le simulacre du substitut » dans GUILLEMARD, Denis (dir.). Du moulage au fac-similé, diffusion du patrimoine et conservation préventive. [Ve Journéesdébats organisées par le DESS de conservation préventive de l'Université de Paris I, 10 et 11 mai 2001]. Paris : ARAAFU, avril 2002, p. 10.

35. - De CONTENSON, Marie-Laure. "Sources documentaires et rôle pédagogique». Dans COLLECTIF. Moulages, copies, facsimilés. [Actes des IXe Journées des restaurateurs en archéologie, bulletin de liaison ${ }^{\circ} 11$ ]. Paris : CNRS Centre d'étude des peintures murales romaines, 1994, p. 67. Voir les autres articles de ce numéro de In situ.

36. - DELOCHE, Bernard. « Et si le substitut valait plus que l'original ». Dans GUILLEMARD, Denis (dir.). Du moulage au fac-similé, diffusion du patrimoine et conservation préventive. [Ve Journées-débats organisées par le DESS de conservation préventive de l'Université de Paris I, 10 et 11 mai 2001]. Paris : ARAAFU, avril 2002, p. 18.

37. - D'après le Livre d'or du musée.

38. - Catalogue de l'exposition: EGGEBRECHT, Eva, EGGEBRECHT, Arne. Ägyptens Aufstieg zur Weltmacht. Ausstellung im Roemer und Pelizaeus Museum, Hildesheim, 1987. Mainz : P. von Zabern, 1987.

39. - EGGEBRECHT, Arne et alii. Ägypten : Götter, Gräber und die Kunst, 4000 Jahre Jenseitsglaube. Band II : Das Grab des Sennefer. [Katalog zur Austellung : Schlossmuseum Linz 9. April Bis 28. September, 1989]. Linz : Oberösterreichisches Landesmuseum, 1989, p. 77.

40. - GUILLEMARD, Denis. «Fidélité et trahison : le simulacre du substitut » dans GUILLEMARD, Denis (dir.). Du moulage au fac-similé, diffusion du patrimoine et conservation préventive. [Ve Journéesdébats organisées par le DESS de conservation préventive de l'Université de Paris I, 10 et 11 mai 2001]. Paris : ARAAFU, avril 2002, p. 13.

41. - Giovanni Battista Belzoni avait découvert la tombe de Séthi $\mathrm{I}^{\mathrm{er}}$ en octobre 1817. Les artistes présents dans l'expédition, en particulier J. Ricci, ont fait des relevés en couleurs de tous les décors de la tombe. C'est à partir de ces dessins qu'a pu être produit un fac-similé. COLLECTIF. Immortal Pharaoh : the Tomb of Thutmose III. [Exposition Edimbourg, City Art Centre, $1^{\text {st }}$ October 2005 - 8 January 2006]. Madrid ; Copenhague : Factum Arte ; United Exhibits Group, 2005, p. 185.

42. - PEARCE, Susan M. "Giovanni Battista Belzoni's exhibition of the re-constructed tomb of Pharaoh Seti I in $1821 »$. Journal of the History of Collections, 2000, n¹2, p. 109.

43. - De CONTENSON, Marie-Laure. "Sources documentaires et rôle pédagogique». Dans COLLECTIF. Moulages, copies, facsimilés. [Actes des IXe Journées des restaurateurs en archéologie, bulletin de liaison $\left.n^{\circ} 11\right]$. Paris : CNRS, Centre d'étude des peintures murales romaines, 1994, p. 69. Voir les autres articles de ce numéro de In situ.

44. - Le fac-similé du Mans a été conservé dans des réserves externes au musée dans la ville du Mans, de 1988 à 2001. Celui d'Hildesheim a été conservé dans des réserves à Berlin gérées par une société privée, de 1988 à 2002. Dans les deux cas, les fac-similés étaient quelquefois prêtés à des musées pour des expositions temporaires.

45. - Selon Panofsky, le fac-similé est une «bonne reproduction " s'il traduit l'intention de l'œuvre d'art sans être forcément une illusion de l'original. PANOFSKY, Erwin. «Original et reproduction en fac-similé (Original und Faksimilereproduktion, 1930)». Les cahiers du Musée National d'art moderne, 1995, n53, p. 46. 
46. - L'espace couleur $\mathrm{L}^{*} \mathrm{a}^{*} \mathrm{~b}^{*}$ est actuellement l'un des plus utilisés pour mesurer la couleur des objets. $\mathrm{L}^{*}$ indique la clarté tandis que $\mathrm{a}^{*}$ et $\mathrm{b}^{*}$ sont les coordonnées de chromaticité $+\mathrm{a}^{*}$ va vers le rouge, $-a^{*}$ vers le vert, $+b^{*}$ va vers le jaune et $-b^{*}$ vers le bleu. Le centre du diagramme de référence est achromatique.

47. - Les informations sont tirées du rapport de restauration, consulté avec l'aimable autorisation du Dr Bettina Schmitz et de Dorothea Lindemann (musée Roemer et Pelizaeus d'Hildesheim) : BREIDENSTEIN, Petra. Dokumentation: Restaurierung Sennefer-Grabkammer Rekonstruktion im November 1998, p. 1-2.

48. - Voir le même rapport de restauration, p. 3-6.

49. - LAVÉDRINE, Bertrand. La conservation des photographies. Paris: Presses du CNRS, 1990. Institut international de conservation des œuvres historiques et artistiques. Section française. Préserver les objets de son patrimoine: précis de conservation préventive. Bruxelles: Mardaga, 2001, p. 201.

50. - SARRET, Françoise et Jean-Pierre, BRIEZ, Serge et Dominique. "La création de nouvelles images du patrimoine et leur diffusion au public: l'archéosynthèse de la "tombe aux vignes", tombeau de Sennefer ». Dans TEFNIN, Roland (éd.). La peinture égyptienne ancienne, un monde de signes à préserver. Monumenta aegyptiaca, 7. [Actes du colloque international, Bruxelles, 1994]. Bruxelles : Fondation égyptologique Reine Élisabeth, 1997, p. 143-149.

\section{RÉSUMÉS}

La tombe de Sennefer, sur la rive gauche du Nil, en face de Louxor, sur la colline de Cheikh Abd el-Gourna, fait partie d'un ensemble patrimonial menacé. L'état de conservation exceptionnel de ses peintures et l'originalité de l'iconographie font la célébrité de celle que l'on appelle communément «la tombe aux vignes». L'ouverture du caveau au public est cependant une importante source de dégradations. La fermeture de la tombe et son remplacement par un facsimilé sont envisageables. Le caveau de Sennefer est d'ailleurs l'un des seuls monuments thébains à avoir fait l'objet de reconstitutions grandeur nature en fac-similé, actuellement exposées au musée de Tessé du Mans et au musée Roemer et Pelizaeus d'Hildesheim.

The tomb of Sennefer, located on the west bank of the Nile, opposite to Luxor, on the hill of Sheikh Abd el-Qurna, belongs to an endangered heritage complex. The exceptional state of conservation of the paintings and its original iconography make this tomb famous : it is also known as the "Tomb of the Vineyards". However, opening the burial chamber to visitors causes damages. Closing down the tomb and replacing it by a facsimile could be conceivable. The burial chamber of Sennefer is, for that matter, one of the only Theban monuments to have been reproduced with full-size facsimiles, currently displayed at the musée de Tessé in Le Mans (France) and at the Roemer-und-Pelizaeus Museum, in Hildesheim (Germany). 
INDEX

Mots-clés : Égypte, Thèbes, Vallée des Nobles, Cheikh Abd el-Gourna, tombe de Sennefer, conservation préventive, fac-similé, Musée de Tessé du Mans, Musée Roemer et Pelizaeus d'Hildesheim

Keywords : Egypt, Theban Necropolis, Tombs of the Nobles, Sheikh Abd el-Qurna, tomb of Sennefer, preventive conservation, facsimile, Musée de Tessé in Le Mans, Roemer- und PelizaeusMuseum, in Hildesheim

\section{AUTEUR}

\section{LAËTITIA MAGGIO}

conférencière nationale, diplômée du deuxième cycle de l'École du Louvre (égyptologie) laetitia.maggio@gmail.com 